\title{
Important classes of reactions for the proactive and reactive resource-constrained project scheduling problem *
}

\author{
Morteza Davari ${ }^{\ddagger \dagger *}$ and Erik Demeulemeester ${ }^{\dagger}$
}

\begin{abstract}
The proactive and reactive resource-constrained project scheduling problem (PR-RCPSP), that has been introduced recently (Davari and Demeulemeester, 2017), deals with activity duration uncertainty in a very unique way. The optimal solution to an instance of the PR-RCPSP is a proactive and reactive policy (PR-policy) that is a combination of a baseline schedule and a set of required transitions (reactions). In this research, we introduce two interesting classes of reactions, namely the class of selection-based reactions and the class of buffer-based reactions, the latter in fact being a subset of the class of selection-based reactions. We also discuss the theoretical relevance of these two classes of reactions. We run some computational results and report the contributions of the selection-based reactions and the buffer-based reactions in the optimal solution. The results suggest that although both selection-based reactions and buffer-based reactions contribute largely in the construction of the optimal PR-policy, the contribution of the buffer-based reactions is of much greater importance. These results also indicate that the contributions of non-selectionbased reactions (reactions that are not selection-based) and selection-but-not-buffer-based reactions (selection-based reactions that are not buffer-based) are very limited.
\end{abstract}

\section{Introduction}

In the recent project scheduling literature, many papers deal with activity duration uncertainty in a resource-constrained project scheduling environment. The methodologies used in these papers often fall into two categories: stochastic scheduling and proactive and reactive scheduling. In the following, a brief review of these papers is given.

In stochastic scheduling, the objective is to minimize the expected makespan while activity durations are stochastic. In such an environment, solutions are policies rather than schedules

\footnotetext{
* This is a draft of an article accepted for publication in Annals of Operations Research.

$\ddagger$ CODeS, Department of Computer Science \& imec-ITEC, KU Leuven KULAK, Etienne Sabbelaan 53 - box 7659 , 8500 Kortrijk (Belgium)

${ }^{\dagger}$ Research Center for Operations Management, Department of Decision Sciences and Information Management, Faculty of Business and Economics, KU Leuven, Naamsestraat 69, 3000 Leuven (Belgium)

${ }^{*}$ Corresponding Author, morteza dot davari at kuleuven dot be
} 
because activity durations are not known in advance and thus activity starting times cannot be decided. A policy is a set of decision rules that determines certain reschedulings at certain decision moments. Different classes of policies have been considered in the literature (Ashtiani et al., 2011; Ballestín and Leus, 2009; Ballestín, 2007; Möhring et al., 1984, 1985; Stork, 2001), some of which will be briefly addressed in the following, namely the class of priority policies (also called resource-based policies (Ashtiani et al., 2011; Fang et al., 2015)), the class of early start policies (Radermacher, 1981), the class of (linear) pre-selective policies (Stork, 2001), the class of activity-based policies (also referred to as job-based policies (Stork, 2001)) and the class of pre-processor policies (Ashtiani et al., 2011; Rostami et al., 2017). These types of policies are considered as open-loop policies (based on the terminology of optimal control theory) as they are static in nature and are not updated during real-time execution.

Alternatives to open-loop policies are closed-loop policies. Closed-loop policies are dynamic in nature and are often obtained in a sequential fashion via dynamic programming (Bertsekas, 2007). Their dynamic and adaptive nature enables them to take advantage of the information that becomes available during the execution and between decision moments. There are a number of papers that focus on closed-loop policies: Choi et al. (2004) propose a number of systematic approximations (i.e., dynamic programming in heuristically confined state spaces) and Li and Womer (2015) develop efficient approximate dynamic programming algorithms based on look-ahead and look-back approximation architectures to obtain quality closed-loop policies. Moreover, Creemers (2015) develops a very efficient dynamic programming-based approach to obtain the best policy for instances where activity durations are exponentially distributed or can be approximated with a phase-type distribution.

Proactive and reactive scheduling consists of two stages. In the first stage, a schedule, which is called the baseline schedule and is as robust ${ }^{1}$ as possible against a certain type of uncertainty, is generated. In the second stage, for each conflict in the ongoing schedule a rescheduling (reaction) occurs. A conflict refers to the situation where the schedule is no longer feasible. Among many papers that deal with uncertainty in RCPSP using proactive and reactive scheduling, we cite Leus (2003) who investigates the construction of stable plans from both a theoretical and a methodological point of view, Van de Vonder et al. (2007) who classify many proactive procedures and reactive strategies and compare the performances of different combinations of a proactive approach and a reactive strategy, Van de Vonder et al. (2008) who propose a number of heuristic methods to construct stable/proactive schedules, Davari and Demeulemeester (2016) who exploit chance-constrained programming as a way of constructing proactive schedules, and finally Davari and Demeulemeester (2017) who introduce four Markov decision processes to solve the proactive and reactive RCPSP.

Although the traditional proactive and reactive procedures are very popular in the lierature (we refer the interested readers to the first chapter of Davari (2017) for an extensive review of such papers), we notice that the authors often forgot two important aspects: firstly, they ignore the impact of the choice of the reactive scheduling policy on the optimality of the baseline schedule and secondly they simply assume that the number of reactions does not have any effect on the robustness of the baseline schedule and on that of the reactive policy. In a previous research (Davari and Demeulemeester, 2017), we introduced the proactive and reactive resource-constrained project scheduling problem (PR-RCPSP) in which both aforementioned forgotten aspects have been addressed and the goal is to find an optimal proactive and reactive policy (PR-policy). A PR-policy is described by a set of decision rules that dictate certain transitions among schedules. In this

\footnotetext{
${ }^{1}$ Robustness refers to the ability of tolerating variabilities that may affect the feasibility of a schedule.
} 
problem setting, we consider a reaction as the transition from one schedule to another schedule. It is worth mentioning that PR-polices are very related and similar to closed-loop policies since both are dynamic in nature and are obtained through dynamic programming recursions. However, they are also different in a sense that PR-policies are defined to consider some degrees of robustness in their decision making, which suits the most the proactive and reactive scheduling problems.

Apart from addressing the two forgotten aspects, there are two other points that make PRRCPSP very interesting in comparison with its traditional alternatives, both of which are thoroughly discussed in Davari and Demeulemeester (2017) and briefly discussed in the following. First, to formulate PR-RCPSP, we took advantage of a new way of reactions (i.e., transitions among schedules), which enabled us to find better quality solutions than those provided by the traditional approaches. Note that the detailed quantitative results to support such a claim have been provided in Davari and Demeulemeester (2017). Second, we argued that PR-policies are more informative and more beneficial (from both a practical and a managerial point of view) than the solution representations introduced in the literature.

While in Davari and Demeulemeester (2017) we mainly focused on formulating and solving the PR-RCPSP as efficiently as possible, our main goal in this paper is to investigate different classes of reactions and to evaluate their contributions in 'optimal'2 PR-policies. In this paper, we

1. introduce two important classes of reactions, namely the class of selection-based reactions and the class of buffer-based reactions. We also prove that the class of buffer-based reactions is a subclass of the class of selection-based reactions,

2. show that determining whether or not a reaction is selection-based can be done in polynomial time and we provide an (in the worst-case) exponential-time algorithm that determines whether or not a reaction is buffer-based,

3. and report via computational results that buffer-based reactions contribute the most in constructing optimal PR-policies.

The remainder of this paper is structured as follows. In Section 2, we provide a brief introduction of the PR-RCPSP from Davari and Demeulemeester (2017) and its dynamic-programming-based solution methods. In Section 3, we introduce the class of selection-based and the class of bufferbased reactions. Then, we propose an exponential-time algorithm to determine whether or not a reaction is buffer-based. After that, we report and discuss the computational results in Section 5 and Section 6. Finally, we summarize and conclude in Section 7.

\section{The PR-RCPSP}

Let us briefly describe the characteristics of the PR-RCPSP. Each instance of this problem consists of a set $N=\{0,1, \ldots, n+1\}$ of activities where activities 0 and $n+1$ are the dummy start and dummy end activities, a set $\mathcal{R}$ of resource constraints and a set $E=\{(i, j) \mid i, j \in N\}$ of precedence constraints among activities. The transitive closure of $E$ is denoted by $T(E)$ and the transitive reduction of $E$ is denoted by $\bar{T}(E)$.

\footnotetext{
${ }^{2}$ Note that the models introduced in Davari and Demeulemeester (2017) only find the optimal PR-policies over different subsets of PR-policies. Finding a globally optimal PR-policy is computationally intractable, although, it is theoretically achievable.
} 
Each activity $i \in N \backslash\{0, n+1\}$ has a non-zero independent stochastic integer duration $\tilde{p}_{i}$, where $p_{i}^{\min } \leq \tilde{p}_{i} \leq p_{i}^{\max }$ and which follows a discrete distribution. Notice that $\tilde{p}_{0}=\tilde{p}_{n+1}=0$. We assume that the realized duration of the activity is only known when the execution of the activity is completed. The vector $\tilde{\mathbf{p}}=\left(\tilde{p}_{0}, \tilde{p}_{1}, \ldots, \tilde{p}_{n+1}\right)$ can be represented by a finite supporting set $\mathfrak{P}=\left\{\mathbf{p}^{l}, \ldots, \mathbf{p}^{|\mathfrak{P}|}\right\}$ of realizations where each realization $\mathbf{p}^{l}$ is represented by a vector $\mathbf{p}^{l}=$ $\left(p_{0}^{l}, p_{1}^{l}, \ldots, p_{(n+1)}^{l}\right) \in \mathfrak{P}$ of realized durations. Each activity $i$ requires $r_{i k}$ units of resource type $k \in \mathcal{R}$ during its processing time. The resource availability of resource type $k$ is denoted by $R_{k}$. Also, each pair $(i, j) \in E$ indicates that activity $j$ cannot be started before activity $i$ is completed.

Solutions in the PR-RCPSP are PR-policies, which is denoted by П. At each decision moment, some information becomes known. This information defines the state of the execution. Each decision in PR-policy $\Pi$ is associated with a certain state of execution. A PR-policy $\Pi$ not only determines reactions, but also selects one schedule as the baseline schedule.

The cost of a PR-policy consists of two parts: the cost of its baseline schedule that is a function of its baseline schedule's activity starting times and the expected cost of all its induced reactions. The cost of a reaction consists on the one hand of a fixed reaction cost and on the other hand of a non-fixed cost that can be a function of the associated starting time deviations. The objective of our introduced proactive and reactive resource-constrained project scheduled problem is to find the policy with the minimum cost. Let $\pi\left(\tilde{\mathbf{p}}=\mathbf{p}^{l}\right)$ represent the probability of occurrence of realization $\mathbf{p}^{l}$. The PR-RCPSP can be formulated as follows:

$$
\min _{\Pi \in \Pi} \sum_{l=1}^{|\mathfrak{P}|} \pi\left(\tilde{\mathbf{p}}=\mathbf{p}^{l}\right)\left(w_{b} \times s_{n+1}^{[0]_{\Pi}}+\sum_{k=1}^{\nu_{\Pi, l}}\left(\sum_{i \in U\left(\mathbf{s}^{\left.[k-1]_{\Pi, l}, t_{k}\right)}\right.} w_{i k}\left|s_{i}^{[k]_{\Pi, l}}-s_{i}^{[k-1]_{\Pi, l}}\right|+w_{r}\right)\right)
$$

where $\Pi$ represent the set of all PR-policies, $w_{b} \geq 0$ is the cost per unit time for the completion time of the baseline schedule, $w_{r} \geq 0$ is the fixed cost incurred with each reaction, $\mathbf{s}^{[0]_{\Pi}}$ is the baseline schedule selected by PR-policy $\Pi, \mathbf{s}^{[k] \Pi, l}$ is the schedule to which we react as the $k$ th reaction under PR-policy $\Pi$ and realization $\mathbf{p}^{l}$, and $w_{i k} \geq 0$ is the stability cost of activity $i$ in the $k$ th reaction.

While finding the optimal PR-policy to the PR-RCPSP seems to be out of reach (computationally intractable), we proposed, in Davari and Demeulemeester (2017), four Markov decision processes (Models 1-4) to obtain the best ('optimal') PR-policies within different subsets (classes) of PR-policies. In the remainder of this section, we very briefly discuss key elements of two of these models, namely Models 1 and 3. Notice that in the computational experiments of this paper, we use Model 3 to evaluate the contributions of our introduced classes of reactions.

\section{$2.1 \quad$ Model 1}

Model 1, which is a Markov decision process, solves the following problem:

$$
\min _{\Pi \in \Pi_{1}} \sum_{l=1}^{|\mathfrak{P}|} \pi\left(\tilde{\mathbf{p}}=\mathbf{p}^{l}\right)\left(w_{b} \times s_{n+1}^{[0]_{\Pi}}+\sum_{k=1}^{\nu_{\Pi, l}}\left(\sum_{i \in U\left(\mathbf{s}^{\left.[k-1]_{\Pi, l}, t_{k}\right)}\right.} w_{i k}\left|s_{i}^{[k]_{\Pi, l}}-s_{i}^{[k-1]_{\Pi, l}}\right|+w_{r}\right)\right)
$$

where $\boldsymbol{\Pi}_{1}$ is the set of all PR-policies that only include schedules from a given set $\mathbf{S}$ that is a subset of $\mathcal{S}$ (the set of all schedules). Obviously, if we choose $\mathbf{S}=\mathcal{S}$ then $\boldsymbol{\Pi}_{1}=\boldsymbol{\Pi}$ and the above formulation is equivalent to the PR-RCPSP. However, such a choice is impractical as the number of states in our model will explode. Therefore, we choose $\mathbf{S}$ as a much smaller subset of $\mathcal{S}$ for practical 
reasons. We acknowledge that the choice of the set $\mathbf{S}$ has a big influence on the performance of our introduced models.

States in Model 1 consist of some information regarding the status of execution. this information includes the current schedule, the current time, the set of ongoing activities and the number of reactions occurred so far. Note that by knowing the current schedule and the set of ongoing activities, one can determine the sets of finished and not-yet-started activities.

Once we arrive at a state that is infeasible, we must transit to a feasible state. To verify whether such a transition is valid, we must guarantee 1- that the sets of finished and ongoing activities are the same for the two states; 2- that the set of not-yet-started activities is the same for the two states; 3 - the current execution times are the same for the two states and 4- that the starting times of all finished and ongoing activities are identical for the two schedules.

\section{$2.2 \quad$ Model 3}

Model 3 is a Markov decision process that solves the following problem:

$$
\min _{\Pi \in \Pi_{3}} \sum_{l=1}^{|\mathfrak{P}|} \pi\left(\tilde{\mathbf{p}}=\mathbf{p}^{l}\right)\left(w_{b} \times s_{n+1}^{[0]_{\Pi}}+\sum_{k=1}^{\nu_{\Pi, l}}\left(\sum_{i \in U\left(\mathbf{s}^{\left.[k-1]_{\Pi, l}, t_{k}\right)}\right.} w_{i k}\left|s_{i}^{[k]_{\Pi, l}}-s_{i}^{[k-1]_{\Pi, l}}\right|+w_{r}\right)\right)
$$

where $\boldsymbol{\Pi}_{3}$ is a larger subset of $\boldsymbol{\Pi}$ than $\boldsymbol{\Pi}_{1}$. We define $\boldsymbol{\Pi}_{3}$ as the set of all PR-policies that are constructed by the schedules in $\mathbf{S}$ and allowing cut transitions. In a cut transition, we ignore the first, the third and the fourth conditions of the last paragraph in Section 2.1. This would allow Model 3 to perform more flexible reactions and thus provide better approximated solutions to the PR-RCPSP than those provided by Model 1 given the same set of schedules $\mathbf{S}$. Note that the size of $\boldsymbol{\Pi}_{3}$ and the quality of its PR-policies are indirectly affected by the given set $\mathbf{S}$. For a more detailed explanation of Model 3 (including a more accurate definition of a cut transition), we strongly advise interested readers to read Section 3.3 in Davari and Demeulemeester (2017).

\section{Two important classes of reactions}

In this section, we study two classes of reactions, namely the class of selection-based reactions and the class of buffer-based reactions. Both selection-based reactions and buffer-based reactions are based on sufficient selections. We first define what is a sufficient selection in Section 3.1, and then introduce the concepts of selection-based reactions and buffer-based reactions in Sections 3.2 and 3.3 , respectively.

Our initial motivation to introduce these two classes of reactions was the fact that we expected that those reactions among schedules which are resulting from the same sufficient selections are often selected in the optimal PR-policy. Therefore, the ultimate objective of this paper is to see what percentage of reactions in an optimal PR-policy are selection-based and what percentage of reactions in an optimal PR-policy are buffer-based.

\subsection{Sufficient selection}

A set $F S$ of activities is a forbidden set if $E \cap(F S \times F S)=\emptyset$ and $\exists k \in \mathcal{R}: \sum_{i \in F S} r_{i k}>R_{k}$. A forbidden sets $F S$ is minimal if for every $i \in F S$, the set $F S \backslash\{i\}$ is not a forbidden set. We 


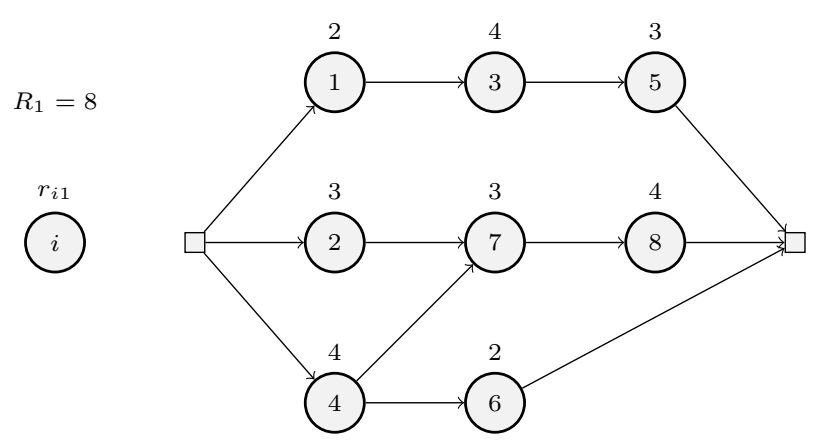

Figure 1: The precedence network for the example project.

define $\mathscr{F}(\cdot)$ as the set of all minimal forbidden sets with $\cdot$ being a partial order among activities. For instance, given the set $E$ of precedence relations among activities, $\mathscr{F}(E)$ is the set of minimal forbidden sets. The concept of forbidden sets was first introduced by Igelmund and Radermacher (1983).

One may use extra resource arcs to eliminate all minimal forbidden sets. Similar ideas have been implemented to address resource incompatibilities in both deterministic and stochastic resourceconstrained project scheduling problems (e.g., Alvarez-Valdes and Tamarit, 1993; Artigues et al., 2013; Leus, 2011a,b). Let us define $X \subset N \times N \backslash T(E)$ as a set of pairs where each pair represents a resource arc. We assume that $X$ is a strict partial order (abbreviated by sp-order) on $N$ (i.e., irreflexible and transitive). For each $(i, j) \in X$, the completion time of activity $i$ must be smaller than or equal to the starting time of activity $j$. We follow Leus (2011b) to call $X$ a selection.

Definition 1 (Sufficient selection). A selection $X$ is called sufficient if and only if $G(N, E \cup X)$ is acyclic and $\mathscr{F}(E \cup X)=\emptyset$.

Example. Consider the instance provided as follows. The activity on node (AoN) representation of this instance is given in Figure 1. Given the deterministic vector of durations $\mathbf{p}=$ $\{0,2,7,4,4,8,6,4,2,0\}$, an optimal solution $\mathbf{s}^{*}=\{0,0,2,4,0,7,7,9,13,15\}$ for the deterministic counterpart with makespan 15 has been computed by RESCON software ${ }^{3}$.

The instance's data can be found in Figure 1 and Tables 1 and 2. Note that this instance is used in all examples in this paper. The AON representation of the precedence relations among the activities for this instance is shown in Figure 1. For this instance, the set of minimal forbidden sets is

$$
\mathscr{F}(E)=\{\{1,2,4\},\{2,3,4\},\{2,3,6\},\{2,4,5\},\{3,6,7\},\{3,6,8\},\{5,6,8\}\} .
$$

Consider selection $X_{1}=\{(4,2),(3,6),(6,8)\}$. Let us include these arcs into the precedence network. The network in Figure 2 results. We notice that $X_{1}$ is a sufficient selection because it suffices to eliminate all minimal forbidden sets (i.e., $\mathscr{F}\left(E \cup X_{1}\right)=\emptyset$ ).

It is worth mentioning that, if a selection $X$ is sufficient, then the induced early-start schedule for every given $\mathbf{p} \in \mathfrak{P}$, which is denoted by $E S(X, \mathbf{p})$, is feasible. However, we argue that the reverse

\footnotetext{
${ }^{3} \mathrm{RESCON}$ is an educational software for the deterministic resource-constrained project scheduling problem (http://www.econ.kuleuven.be/rescon/)
} 


\begin{tabular}{c|c|ccc|c}
\hline & $\hat{p}_{i}$ & \multicolumn{3}{|c}{$\pi\left(\tilde{p}_{i}=\hat{p}_{i}+\epsilon\right)$} & $w_{i, 0}$ \\
& & $\epsilon=-1$ & $\epsilon=0$ & $\epsilon=+1$ & \\
\hline$\tilde{p}_{0}$ & 0 & 0 & 1 & 0 & - \\
$\tilde{p}_{1}$ & 2 & 0.4 & 0.4 & 0.2 & 4 \\
$\tilde{p}_{2}$ & 7 & 0.3 & 0.5 & 0.2 & 4 \\
$\tilde{p}_{3}$ & 3 & 0 & 0.6 & 0.4 & 7 \\
$\tilde{p}_{4}$ & 4 & 0.1 & 0.5 & 0.4 & 1 \\
$\tilde{p}_{5}$ & 8 & 0.2 & 0.8 & 0 & 4 \\
$\tilde{p}_{6}$ & 6 & 0.4 & 0.6 & 0 & 1 \\
$\tilde{p}_{7}$ & 4 & 0.5 & 0.5 & 0 & 1 \\
$\tilde{p}_{8}$ & 2 & 0 & 0.7 & 0.3 & 1 \\
$\tilde{p}_{9}$ & 0 & 0 & 1 & 0 & 38
\end{tabular}

Table 1: The distribution of activity durations and the weights of the activities for the first reaction.

\begin{tabular}{c|cccccccccc}
\hline & \multicolumn{1}{|c}{$\mathbf{s}^{k}$} & $\mathbf{s}^{k}$ & $\mathbf{s}^{7}$ & $\mathbf{s}^{8}$ & $\mathbf{s}^{9}$ & $\mathbf{s}^{10}$ \\
\hline$s_{0}^{k}$ & 0 & 0 & 0 & 0 & 0 & 0 & 0 & 0 & 0 & 0 \\
$s_{1}^{k}$ & 0 & 0 & 0 & 0 & 0 & 0 & 0 & 0 & 0 & 0 \\
$s_{2}^{k}$ & 1 & 1 & 0 & 1 & 5 & 0 & 7 & 4 & 2 & 7 \\
$s_{3}^{k}$ & 3 & 3 & 4 & 4 & 3 & 3 & 3 & 3 & 5 & 5 \\
$s_{4}^{k}$ & 0 & 0 & 4 & 0 & 0 & 7 & 0 & 0 & 0 & 9 \\
$s_{5}^{k}$ & 6 & 6 & 7 & 7 & 7 & 7 & 7 & 7 & 9 & 14 \\
$s_{6}^{k}$ & 6 & 6 & 7 & 7 & 7 & 12 & 5 & 7 & 9 & 14 \\
$s_{7}^{k}$ & 7 & 8 & 7 & 8 & 12 & 12 & 14 & 12 & 11 & 15 \\
$s_{8}^{k}$ & 11 & 13 & 13 & 12 & 15 & 15 & 17 & 15 & 15 & 20 \\
$s_{9}^{k}$ & 13 & 15 & 15 & 15 & 17 & 18 & 19 & 18 & 18 & 23
\end{tabular}

Table 2: A given set $\mathbf{S}$ for the example project.

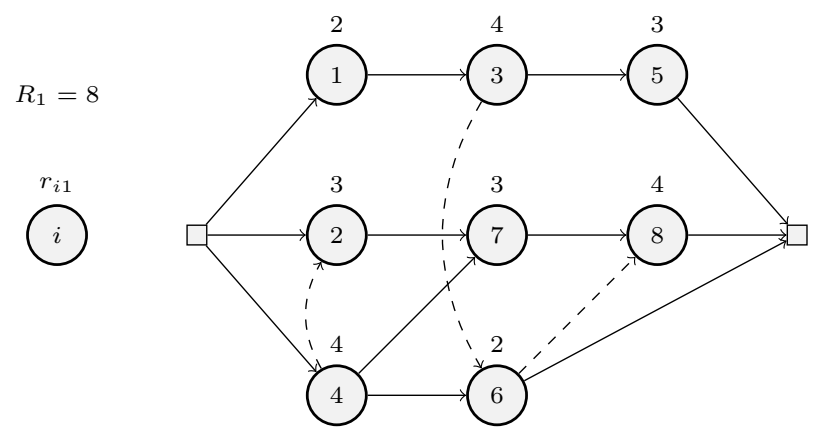

Figure 2: The precedence network for the example project including extra (dashed) arcs for $X_{1}$.

relation is not necessarily true. In other words, the fact that $E S(X, \mathbf{p})$ is feasible for each $\mathbf{p} \in \mathfrak{P}$ is not adequate to conclude that $X$ is sufficient. In the following, we provide a counterexample that supports the mentioned argument. 
Example. Consider the deterministic version of the instance given in the previous example. For this instance, $\mathfrak{P}=\{\hat{\mathbf{p}}\}$ (remember from Table 1 that $\hat{\mathbf{p}}=(0,2,7,3,4,8,6,4,2,0)$ ). Even though the selection $X_{2}=\{(4,2),(3,6)\}$ is not sufficient because $\mathscr{F}\left(E \cup X_{2}\right)=\{\{5,6,8\}\}$, the schedule

$$
E S\left(X_{2}, \hat{\mathbf{p}}\right)=(0,0,4,2,0,5,5,11,13)
$$

is feasible.

In the following, we discuss whether or not an efficient algorithm exists that determines the sufficiency of a selection. The problem of determining whether or not $X$ is sufficient is equivalent to the problem of determining whether or not there is at least one (minimal) forbidden set for the associated instance of the RCPSP with $G(N, E \cup X)$. Both problems are solved by finding the maximum weighted anti-chain in $G(N, E \cup X)$ where weights are the resource requirements. Finding the maximum weighted anti-chain in $G(N, E \cup X)$ is equivalent to finding the maximum weight independent set (or its associated maximum weight clique) in $G(N, E \cup X)$. From Grötschel et al. (1984, Theorem 6.5) we know that there exists a polynomial-time algorithm for any maximum weight clique problem with a perfect graph. Since any partially ordered set including $G(N, E \cup X)$ is a comparability graph (which is a well-known perfect graph), we conclude that there must exist a polynomial algorithm that, given an instance of RCPSP with $G(N, E)$ and a selection $X$, determines whether or not $X$ is sufficient.

A number of polynomial algorithms exist to determine the maximum weighted anti-chain (stable set) in $G(N, E \cup X)$, among which we cite the max-flow-based algorithms described in Leus (2003), Neumann et al. (2003) and Schwindt (2005). Other similar interesting results and algorithms can be found in Cong (1993), Golumbic (1980) and Kaerkes and Leipholz (1977).

\subsection{Selection-based reactions}

Since the activity durations are stochastic, robust schedules usually include buffers. Unlike many buffer insertion techniques in the literature in which buffers are inserted before the start of the activities (e.g., Lambrechts et al., 2008; Mehta and Uzsoy, 1998; Van de Vonder et al., 2008)), we assume that the buffers are inserted after the completion times of the activities. We also assume that buffers require the same resources as their associated activities. There are two motivations behind these assumptions. First, with these two assumptions, we ensure that every vector of durations $\mathbf{p} \in \mathfrak{P}$ associates a vector of buffers $\mathbf{b}$ where $\mathbf{p}=\mathbf{p}^{\min }+\mathbf{b}$. Second, in contrast with the alternative approach, in which buffers are inserted before the start of the activities, this approach produces no 'useless' buffers. When we insert buffers before the start of activity $i$, only the activities that finished immediately before activity $i$ can use such buffers. If the resource requirements of these activities are different from the resource requirements of activity $i$, there are chances that these activities cannot use the inserted buffers, in which case the inserted buffers are considered 'useless'. Alternatively, when we insert buffers after the completion time (according to its minimum duration) of activity $i$, they become beneficial at least for activity $i$ and are never considered 'useless'.

Given any schedule $\mathbf{s}$ and any vector of buffers $\mathbf{b}$, a unique associated sp-order can be constructed as follows. Let $\mathcal{X}_{\mathbf{s}, \mathbf{b}}$ represent the sp-order induced by the pair $(\mathbf{s}, \mathbf{b})$. We define $\mathcal{X}_{\mathbf{s}, \mathbf{b}}$ by $(i, j) \in$ $\mathcal{X}_{\mathbf{s}, \mathbf{b}} \Leftrightarrow s_{i}+p_{i}^{\min }+b_{i} \leq s_{j}$, where $i, j \in N$ with $i \neq j$.

A sufficient selection $X$ is feasible for schedule $\mathbf{s}$ if for some realization $\mathbf{p} \in \mathfrak{P}$ (note that $\mathbf{p}=\mathbf{p}^{\text {min }}+\mathbf{b}$ ), we have $X \subseteq \mathcal{X}_{\mathbf{s}, \mathbf{b}} \backslash T(E)$. Here the sp-order induced by pair $(\mathbf{s}, \mathbf{0})$ becomes interesting because for every vector of buffers $\mathbf{b}$ so that schedule $\mathbf{s}$ is feasible for $\mathbf{p}=\mathbf{p}^{\mathrm{min}}+\mathbf{b}$, the 
following relation holds: $\mathcal{X}_{\mathbf{s}, \mathbf{b}} \subseteq \mathcal{X}_{\mathbf{s}, \mathbf{0}}$. Thus, we infer that a sufficient selection $X$ is feasible for schedule $\mathbf{s}$ if $X \subseteq \mathcal{X}_{\mathbf{s}, \mathbf{0}} \backslash T(E)$.

Definition 2 (Selection-based reaction). A reaction from $\mathbf{s}$ to $\mathbf{s}^{\prime}$ is selection-based if there is a sufficient selection $X$ that is feasible for both $\mathbf{s}$ and $\mathbf{s}^{\prime}$.

The following theorem provides the only necessary and also sufficient condition for a given reaction to be selection-based.

Theorem 1. A reaction from $\mathbf{s}$ to $\mathbf{s}^{\prime}$ is selection-based if and only if $X=\left(\mathcal{X}_{\mathbf{s}, \mathbf{0}} \cap \mathcal{X}_{\mathbf{s}^{\prime}, \mathbf{0}}\right) \backslash T(E)$ is sufficient.

Proof. On the one hand, we argue that $X=\left(\mathcal{X}_{\mathbf{s}, \mathbf{0}} \cap \mathcal{X}_{\mathbf{s}^{\prime}, \mathbf{0}}\right) \backslash T(E)$ is feasible for both $\mathbf{s}$ and $\mathbf{s}^{\prime}$ because $X \subseteq \mathcal{X}_{\mathbf{s}, \mathbf{0}} \backslash T(E)$ and $X \subseteq \mathcal{X}_{\mathbf{s}^{\prime}, \mathbf{0}} \backslash T(E)$. If $X$ is sufficient, then the conditions of Definition 2 are met and the reaction from $\mathbf{s}$ to $\mathbf{s}^{\prime}$ is selection-based.

On the other hand, if the reaction from $\mathbf{s}$ to $\mathbf{s}^{\prime}$ is selection-based, then based on the definition there exists a selection $X^{\prime}$ that is feasible for both $\mathbf{s}$ and $\mathbf{s}^{\prime}$. The immediate conclusion is that the two relations $X^{\prime} \subseteq \mathcal{X}_{\mathbf{s}, \mathbf{0}} \backslash T(E)$ and $X^{\prime} \subseteq \mathcal{X}_{\mathbf{s}^{\prime}, \mathbf{0}} \backslash T(E)$ must hold and therefore

$$
X^{\prime} \subseteq X=\left(\mathcal{X}_{\mathbf{s}, \mathbf{0}} \cap \mathcal{X}_{\mathbf{s}^{\prime}, \mathbf{0}}\right) \backslash T(E)
$$

also holds. It is straightforward to see that since $X^{\prime}$ is sufficient, $X$ must also be sufficient which concludes the proof.

Based on Theorem 1, and as checking the sufficiency of a given selection can be done in polynomial time, determining whether or not a given reaction is selection-based can also be done in polynomial time.

Example. Let us slightly change the instance given in the example of Section 3.2 and construct a slightly different instance. The new instance is exactly the same as the original instance, except that the distribution of the duration of activity 3 is given as follows:

$$
\pi\left(\tilde{p}_{3}=2\right)=0.2, \pi\left(\tilde{p}_{3}=3\right)=0.4 \text { and } \pi\left(\tilde{p}_{3}=4\right)=0.4 .
$$

Therefore, unlike the original instance where $p_{3}^{\min }=3$, in the new instance $p_{3}^{\min }=2$.

Consider the reaction from $\mathbf{s}^{7}$ to $\mathbf{s}^{9}$ for the new instance (the Gantt charts associated with these two schedules are provided in Figure 3).Figure 4(a) represents the associated graph for the transitive reduction of $\mathcal{X}_{\mathbf{s}^{7}, \mathbf{0}}$ and Figure $4(b)$ represents the associated graph for the transitive reduction of $\mathcal{X}_{\mathbf{s}^{9}, \mathbf{0}}$. Figure $4(c)$ depicts the associated graph for the transitive reduction of $\left(\mathcal{X}_{\mathbf{s}^{7}, \mathbf{0}} \cap \mathcal{X}_{\mathbf{s}^{9}, \mathbf{0}}\right)$. As can be noticed in the graph of Figure $4(c), X=\mathcal{X}_{\mathbf{s}^{7}, \mathbf{0}} \cap \mathcal{X}_{\mathbf{s}^{9}, \mathbf{0}} \backslash T(E)$ is sufficient (i.e., $\mathscr{F}(E \cup X)=\emptyset$ ). Since, by definition, $X$ is feasible for both $\mathbf{s}^{7}$ and $\mathbf{s}^{9}$, the reaction from $\mathbf{s}^{7}$ to $\mathbf{s}^{9}$ is a selection-based reaction.

Let us consider the reaction from $\mathbf{s}^{7}$ to $\mathbf{s}^{9}$ for the original instance. In this case, $p_{3}^{\min }=3$ and the arc $(3,6)$ in both graphs of Figures $4(a)$ and $4(c)$ is replaced by $(4,6)$. Therefore, $X$ is no longer sufficient (there exists a forbidden set, namely $\{2,3,6\})$ and the reaction is not selection-based.

\subsection{Buffer-based reactions}

Despite the fact that for every selection-based reaction from $\mathbf{s}$ to $\mathbf{s}^{\prime}$ there is a sufficient selection $X \subseteq\left(\mathcal{X}_{\mathbf{s}, \mathbf{0}} \cap \mathcal{X}_{\mathbf{s}^{\prime}, \mathbf{0}}\right) \backslash T(E)$ that is feasible for both $\mathbf{s}$ and $\mathbf{s}^{\prime}$, there is no guarantee that one can 


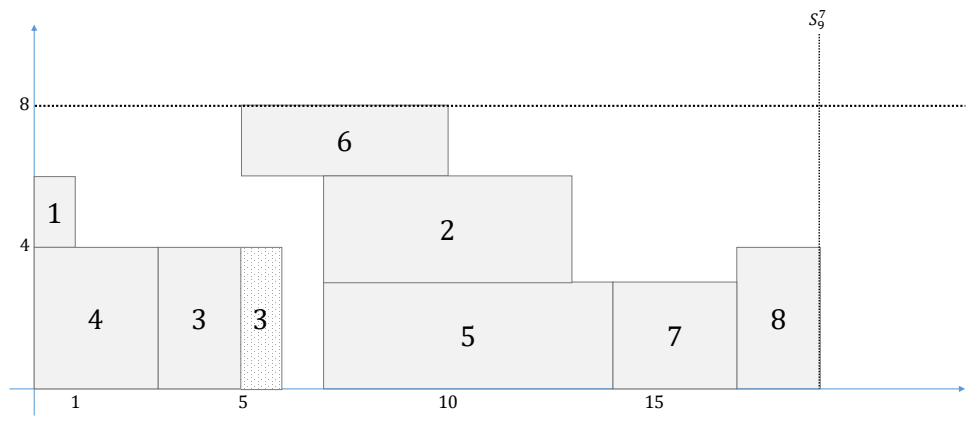

(a) Gantt chart for schedule $\mathbf{s}^{7}$

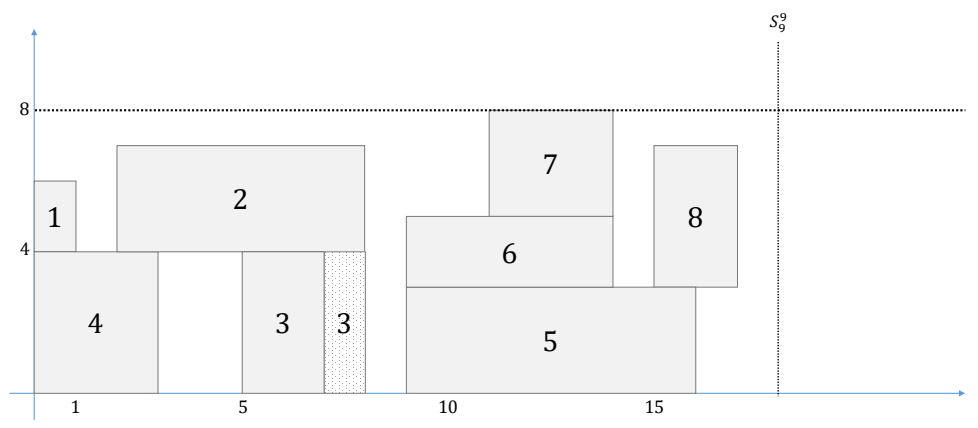

(b) Gantt chart for schedule $\mathbf{s}^{9}$

Figure 3: Gantt charts associated with schedules $\mathbf{s}^{7}$ and $\mathbf{s}^{9}$ in the example of Section 3.2. In order to produce these Gantt charts, the vector of minimum activity durations $\mathbf{p}^{\mathrm{min}}$ is used. Note that the minimum duration of activity 3 equals 2 for the new instance introduced for the example of Section 3.2 and equals 3 for the original instance.

actually construct schedules $\mathbf{s}$ and $\mathbf{s}^{\prime}$ using selection $X$ (we assume that selection $X$ can construct $\mathbf{s}$ if and only if there exists a vector $\mathbf{b}$ such that $\left.\mathbf{s}=E S\left(X, \mathbf{p}^{\min }+\mathbf{b}\right)\right)$. In this subsection, we introduce buffer-based reactions for which there exists a selection that can be used to construct both participating schedules.

A pair $(X, \mathbf{b})$ induces schedule $\mathbf{s}$ if $X$ is sufficient and $\mathbf{s}=E S\left(X, \mathbf{p}^{\min }+\mathbf{b}\right)$. Similarly, a 3 -tuple $\left(X, \mathbf{b}, \mathbf{b}^{\prime}\right)$ induces a pair of schedules $\left(\mathbf{s}, \mathbf{s}^{\prime}\right)$ if $X$ is sufficient, $\mathbf{s}=E S\left(X, \mathbf{p}^{\min }+\mathbf{b}\right)$ and $\mathbf{s}^{\prime}=E S\left(X, \mathbf{p}^{\min }+\mathbf{b}^{\prime}\right)$. By definition, if pair $(X, \mathbf{b})$ induces $\mathbf{s}$, then $X$ is sufficient and $\mathbf{s} \in \mathcal{S}\left(\mathbf{p}^{\min }+\mathbf{b}\right)$ (note that $\mathcal{S}(\mathbf{p})$ represents the set of all feasible solutions for realization $\mathbf{p}$ ). A similar argument is true for every 3 -tuple $\left(X, \mathbf{b}, \mathbf{b}^{\prime}\right)$ and its induced pair $\left(\mathbf{s}, \mathbf{s}^{\prime}\right)$. Notice that while for each 3 -tuple $\left(X, \mathbf{b}, \mathbf{b}^{\prime}\right)$ there is exactly one induced pair of schedules, the reverse relation is rarely true.

Definition 3 (Buffer-based reaction). A reaction from $\mathbf{s}$ to $\mathbf{s}^{\prime}$ is a buffer-based reaction if there exists a 3-tuple $\left(X, \mathbf{b}, \mathbf{b}^{\prime}\right)$ that induces $\left(\mathbf{s}, \mathbf{s}^{\prime}\right)$.

In other words, a buffer-based reaction is a reaction in which a new schedule is created only by changing the vector of buffers (i.e., the associated selection remains the same). 


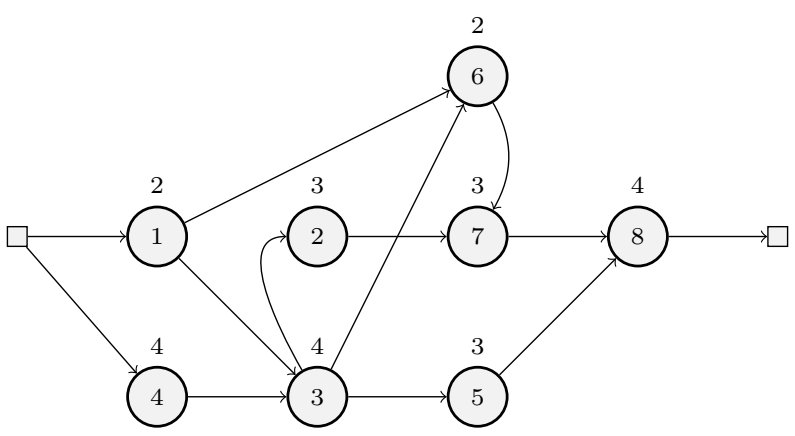

(a) This figure represents $G\left(N, \bar{T}\left(\mathcal{X}_{\mathbf{s}^{7}, \mathbf{0}}\right)\right)$

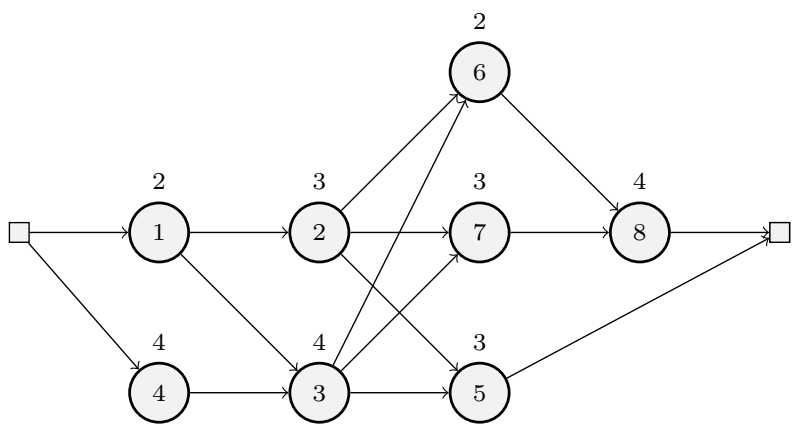

(b) This figure represents $G\left(N, \bar{T}\left(\mathcal{X}_{\mathbf{s}^{9}, \mathbf{0}}\right)\right)$

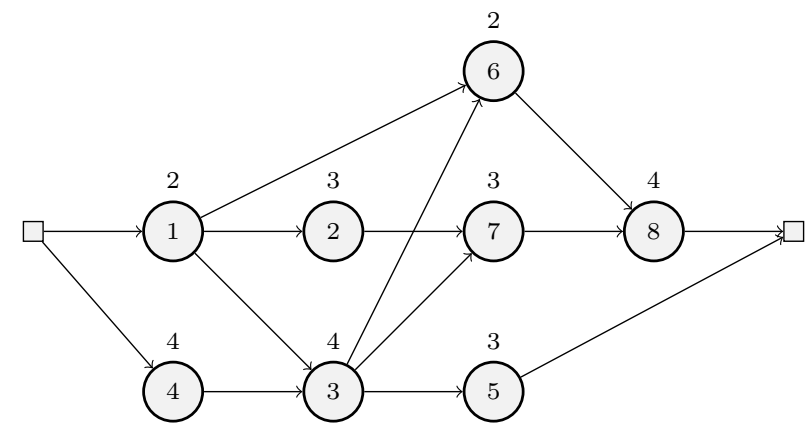

(c) This figure represents $G\left(N, \bar{T}\left(\mathcal{X}_{\mathbf{s}^{7}, \mathbf{0}} \cap \mathcal{X}_{\mathbf{s}^{9}, \mathbf{0}}\right)\right)=G(N, \bar{T}(E \cup X)$

Figure 4: The graphs associated with the example in Section 3.2.

Example. The selection-based reaction from $\mathbf{s}^{7}$ to $\mathbf{s}^{9}$ that has been provided in Section 3.2 is not a buffer-based reaction (see Section 4 in which we provide the reason why this reaction is not buffer-based). In contrast to this reaction, a reaction from $\mathbf{s}^{8}$ to $\mathbf{s}^{5}$ (see Figures $5(a)$ and $\left.5(b)\right)$ is buffer-based because there exists a 3-tuple $\left(X, \mathbf{b}, \mathbf{b}^{\prime}\right)$ with

$$
\begin{aligned}
X & =\{(4,2),(3,6),(6,8)\}, \\
\mathbf{b} & =(0,2,2,1,1,1,1,0,1,0) \text { and } \\
\mathbf{b}^{\prime} & =(0,2,1,1,2,1,1,0,0,0)
\end{aligned}
$$




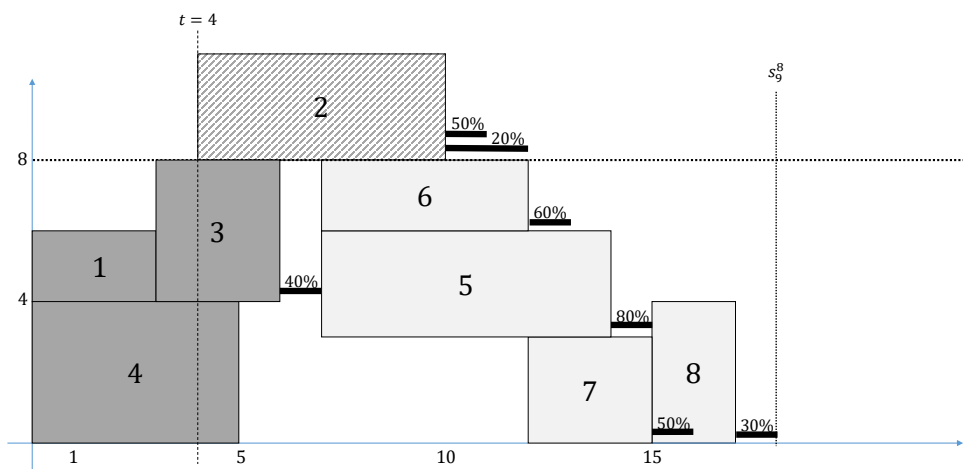

(a) Gantt chart for schedule $\mathbf{s}^{8}$ which becomes infeasible at time 4 for realization $\mathbf{p}^{3}$

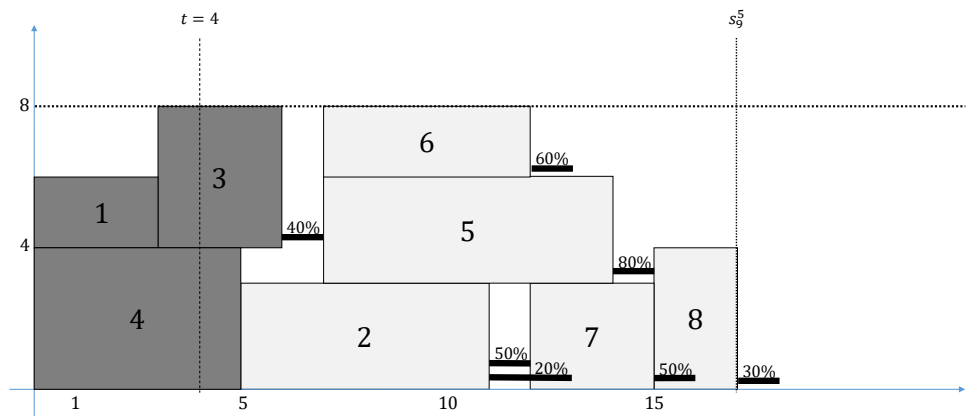

(b) Gantt chart for schedule $\mathbf{s}^{5}$ which is feasible at time 4 for realization $\mathbf{p}^{3}$

Figure 5: A reaction from $\mathbf{s}^{8}$ to $\mathbf{s}^{5}$ at time 4 . The length of each box indicates the minimum possible duration for the associated activity. Other possible durations of an activity are indicated by horizontal bars together with their probabilities of occurrence. Note that at time $t$ the lengths of the associated boxes of the finished activities represent their realized duration. Also notice that the minimum length of the box associated with an ongoing activity cannot be less than or equal to its realized duration so far.

that induces the pair $\left(\mathbf{s}^{8}, \mathbf{s}^{5}\right)$.

Let us define the class of stochastically semi-active schedules. This class consists of schedules, each of which is semi-active for at least one vector of durations. We label these schedules differently than the semi-active schedules since the original concept of semi-active schedules has been defined for deterministic scheduling problems.

Theorem 2. For each schedule $\mathbf{s}$, there exists at least one pair $(X, \mathbf{b})$ for which $\mathbf{s}=E S(X, \mathbf{b})$ if and only if $\mathbf{s}$ is stochastically semi-active.

Proof. On the one hand, if there exists a pair $(X, \mathbf{b})$ for which $\mathbf{s}=E S(X, \mathbf{b})$, then by definition $\mathbf{s}$ is stochastically semi-active. On the other hand, if $\mathbf{s}$ is stochastically semi-active, then there exists at least a vector of durations $\mathbf{p} \in \mathfrak{P}$ for which $\mathbf{s}$ is semi-active. Let $\mathbf{b}=\mathbf{p}-\mathbf{p}^{\min }$ and $X=\mathcal{X}_{\mathbf{s}, \mathbf{b}} \backslash T(E)$. It is straightforward to see that $X$ is sufficient and $\mathbf{s}=E S(X, \mathbf{b})$. 
Based on the definition of buffer-based reactions and also on Theorem 2, if either $\mathbf{s}$ or $\mathbf{s}^{\prime}$ is not stochastically semi-active, the associated reaction cannot be buffer-based. Our (initial) pool generation scheme, however, only generates stochastically semi-active schedules.

Theorem 3. The class of buffer-based reactions is a subset of the class of selection-based reactions.

Proof. If a reaction from $\mathbf{s}$ to $\mathbf{s}^{\prime}$ is buffer-based, then by definition there is a selection $X$ that is sufficient and that is feasible for both schedules $\mathbf{s}$ and $\mathbf{s}^{\prime}$. Therefore this reaction is also selectionbased.

From Theorem 3, we also infer that any reaction that is not selection-based is certainly not buffer-based. However, in general, determining whether or not a reaction from $\mathbf{s}$ to $\mathbf{s}^{\prime}$ is bufferbased is not trivial. We need to either find one associated 3 -tuple $\left(X, \mathbf{b}, \mathbf{b}^{\prime}\right)$ that induces $\left(\mathbf{s}, \mathbf{s}^{\prime}\right)$ or to prove that there exists no such 3 -tuple. We introduce the following decision problem:

Problem BBP

Instance: A PR-RCPSP instance $(N, E, \tilde{\mathbf{p}}, \mathbf{r}, \mathcal{R})$ and a pair $\left(\mathbf{s}, \mathbf{s}^{\prime}\right)$.

Question: Does there exist a 3-tuple $\left(X, \mathbf{b}, \mathbf{b}^{\prime}\right)$ that induces $\left(\mathbf{s}, \mathbf{s}^{\prime}\right)$ ?

If the answer to BBP is 'YES', then the reaction from $\mathbf{s}$ to $\mathbf{s}$ ' is buffer-based. Otherwise, if the answer is 'NO', the reaction is not buffer-based. Currently, the complexity of this problem is still open. Nevertheless, in Section 4, we provide an exponential time algorithm that solves BBP.

\section{An implicit enumeration algorithm to determine buffer- based reactions}

As stated before, if the reaction is not selection-based, then the answer to problem BBP is a definite 'NO'. If however the reaction is selection-based, we must enumerate all 3-tuples $\left(X, \mathbf{b}, \mathbf{b}^{\prime}\right)$ with $X \subseteq\left(\mathcal{X}_{\mathbf{s}, \mathbf{0}} \cap \mathcal{X}_{\mathbf{s}^{\prime}, \mathbf{0}}\right) \backslash T(E)$ and see if at least one of these 3 -tuples satisfies the condition of Definition 3. In the following, we provide an implicit enumeration algorithm.

Consider schedule $\mathbf{s}$ and pair $(X, \mathbf{b})$. Activity $j$ pushes activity $i$ in schedule $\mathbf{s}$ for pair $(X, \mathbf{b})$ if $s_{j}+p_{j}^{\min }+b_{j}=s_{i}$ and $(j, i) \in T(X \cup E)$. Let $\mathcal{A}(\mathbf{s}, X, \mathbf{b})$ be the set of all pushing pairs $(j, i)$ where $j$ pushes $i$ in schedule $\mathbf{s}$ for pair $(X, \mathbf{b})$. The set $\mathcal{A}(\mathbf{s}, X, \mathbf{b})$ is called a complete pushing set if for each $i \in N \backslash\{0\}$ there is at least one $(j, i) \in \mathcal{A}(\mathbf{s}, X, \mathbf{b})$. The following theorem holds.

Theorem 4. $\mathbf{s}=E S\left(X, \mathbf{p}^{\text {min }}+\mathbf{b}\right)$ if and only if $\mathcal{A}(\mathbf{s}, X, \mathbf{b})$ is a complete pushing set.

Proof. If $\mathbf{s}=E S\left(X, \mathbf{p}^{\text {min }}+\mathbf{b}\right)$, then by definition each activity $i \in N \backslash\{0\}$ starts at the completion time of another activity $j \in N \backslash\{n+1\}$ and thus $\mathcal{A}(\mathbf{s}, X, \mathbf{b})$ is a complete pushing set. On the other hand, if $\mathcal{A}(\mathbf{s}, X, \mathbf{b})$ is a complete pushing set, then $s_{j}+p_{j}^{m i n}+b_{j}=s_{i}$ for each $(j, i) \in \mathcal{A}(\mathbf{s}, X, \mathbf{b})$. We also know that $s_{j}+p_{j}^{\text {min }}+b_{j} \leq s_{i}$ for each $(j, i) \in T(X \cup E)$. Therefore, we conclude

$$
s_{i}=\max _{(i, j) \in T(X \cup E)}\left\{s_{j}+p_{j}^{m i n}+b_{j}\right\}
$$

which suffices to infer that $\mathbf{s}=E S\left(X, \mathbf{p}^{\min }+\mathbf{b}\right)$. Note that we assume that the dummy start activity always starts and finishes at time zero. 
Based on Theorem 4, we deduce that if both complete pushing sets $\mathcal{A}(\mathbf{s}, X, \mathbf{b})$ and $\mathcal{A}\left(\mathbf{s}^{\prime}, X, \mathbf{b}^{\prime}\right)$ exist, then a 3 -tuple $\left(X, \mathbf{b}, \mathbf{b}^{\prime}\right)$ that induces $\left(\mathbf{s}, \mathbf{s}^{\prime}\right)$ also exists.

We define a set $B$ of activities $(B \subseteq N \backslash\{0, n+1\})$ that represents all activities that are pushed by the dummy start activity (i.e., all activities that start at time zero in both schedules). We construct an activity list (AL) by sorting all activities in $N \backslash(B \cup\{0\})$ based on the non-decreasing order of their starting times in one of the schedules (for instance the schedule from which we react). As a tie-break rule, the activity with the smaller index is placed before the activity with the larger index. Note that AL is an array with $n-|B|+1$ members and $\mathrm{AL}_{k}$ refers to the activity in the $k$ th position in AL.

We propose a branching tree in which each node (which is denoted by $\mathcal{N}_{u}$ where $u$ is the index of the node, indicating the sequence in which the nodes are visited) represents a pair of partially constructed pushing sets $\left(\mathcal{A}_{u}, \mathcal{A}_{u}^{\prime}\right)$ with $\mathcal{A}_{u}$ being the associated set of pushing pairs for $\mathbf{s}$ and $\mathcal{A}_{u}^{\prime}$ being the associated set of pushing pairs for $\mathbf{s}^{\prime}$. The first two levels are associated with the first activity in the AL, the next two levels are associated with the second activity in the AL and so on. Finally the last two levels are associated with the last activity in the AL. Therefore, this tree consists of $2(n-|B|+1)$ levels. In each odd level of the tree, we add one pushing pair $(j, i)$ to $\mathcal{A}_{u}$ and in each even level, we add one pushing pair $\left(j^{\prime}, i^{\prime}\right)$ to $\mathcal{A}_{u}^{\prime}$ where $i$ and $i^{\prime}$ are the associated activities for the two levels, respectively.

Given any pair of partially constructed pushing sets $\left(\mathcal{A}_{u}, \mathcal{A}_{u}^{\prime}\right)$, we construct an associated 3-tuple $\left(X\left(\mathcal{A}_{u}, \mathcal{A}_{u}^{\prime}\right), \mathbf{b}\left(\mathcal{A}_{u}\right), \mathbf{b}^{\prime}\left(\mathcal{A}_{u}\right)\right)$ with $X\left(\mathcal{A}_{u}, \mathcal{A}_{u}^{\prime}\right)$ being the associated selection and $\mathbf{b}\left(\mathcal{A}_{u}\right)$ and $\mathbf{b}^{\prime}\left(\mathcal{A}_{u}\right)$ being the associated vectors of buffers. In the following, we explain how we compute $X\left(\mathcal{A}_{u}, \mathcal{A}_{u}^{\prime}\right)$, $\mathbf{b}\left(\mathcal{A}_{u}\right)$ and $\mathbf{b}^{\prime}\left(\mathcal{A}_{u}\right)$. Let $X^{a}=\left(\mathcal{X}_{\mathbf{s}, \mathbf{0}} \cap \mathcal{X}_{\mathbf{s}^{\prime}, \mathbf{0}}\right) \backslash T(E)$ be the set of all arcs that are candidates to be included in any selection associated to $\left(\mathcal{A}_{u}, \mathcal{A}_{u}^{\prime}\right)$. By assuming that $j$ pushes $i$ in $\mathbf{s}$ (which is equivalent to adding $(j, i)$ to $\left.\mathcal{A}_{u}\right)$, we also accept that all $\operatorname{arcs}(j, k)$ in $T\left(E \cup X^{a}\right)$ such that $s_{i}>s_{k}$ (note that $\left.s_{j}+p_{j}^{\min }+b_{j}\left(\mathcal{A}_{u}\right)=s_{i}\right)$ are violated in $\mathbf{s}$. The set of all violating arcs that are induced by all pushing pairs in $\mathcal{A}_{u}$ is referred to as $V\left(\mathcal{A}_{u}\right)$. We obtain $V\left(\mathcal{A}_{u}^{\prime}\right)$ in a similar way. The associated selection $X\left(\mathcal{A}_{u}, \mathcal{A}_{u}^{\prime}\right)$ is computed as follows:

$$
X\left(\mathcal{A}_{u}, \mathcal{A}_{u}^{\prime}\right)=X^{a} \backslash\left(V\left(\mathcal{A}_{u}\right) \cup V\left(\mathcal{A}_{u}^{\prime}\right)\right) .
$$

Moreover, for each partially constructed pushing set $\mathcal{A}_{u}$, we compute its associated set of buffers which is denoted by $\mathbf{b}\left(\mathcal{A}_{u}\right)$ as follows:

$$
b_{j}\left(\mathcal{A}_{u}\right)=\left\{\begin{array}{ll}
0 & \text { if } \forall i \in N \backslash\{0, j\},(j, i) \notin \mathcal{A}_{u} \\
s_{i}-s_{j}-p_{j}^{\text {min }} & \text { if } \exists i \in N \backslash\{0, j\},(j, i) \in \mathcal{A}_{u}
\end{array} .\right.
$$

With a similar reasoning, we can obtain $\mathbf{b}^{\prime}\left(\mathcal{A}_{u}\right)$.

Not every possible choice of $\left(\mathcal{A}_{u}, \mathcal{A}_{u}^{\prime}\right)$ is feasible. A pair of pushing sets $\left(\mathcal{A}_{u}, \mathcal{A}_{u}^{\prime}\right)$ is feasible if its associated 3-tuple $\left(X\left(\mathcal{A}_{u}, \mathcal{A}_{u}^{\prime}\right), \mathbf{b}\left(\mathcal{A}_{u}\right), \mathbf{b}^{\prime}\left(\mathcal{A}_{u}\right)\right)$ induces $\left(\mathbf{s}, \mathbf{s}^{\prime}\right)$. In the following, we propose five conditions that must be met to conclude that $\left(\mathcal{A}_{u}, \mathcal{A}_{u}^{\prime}\right)$ is feasible. The proofs for the necessity of these five conditions are given in Theorem 5 .

Condition 1. For every $i, i^{\prime}, j \in N$ such that $(j, i) \in \mathcal{A}_{u}$ and $\left(j, i^{\prime}\right) \in \mathcal{A}_{u}$, the equality $s_{i}=s_{i^{\prime}}$ holds. Also, for every $i, i^{\prime}, j \in N$ such that $(j, i) \in \mathcal{A}_{u}^{\prime}$ and $\left(j, i^{\prime}\right) \in \mathcal{A}_{u}^{\prime}$, the equality $s_{i}^{\prime}=s_{i^{\prime}}^{\prime}$ holds.

Condition 2. $\mathbf{s}$ and $\mathbf{s}^{\prime}$ are resource feasible for $\mathbf{p}^{\min }+\mathbf{b}\left(\mathcal{A}_{u}\right)$ and $\mathbf{p}^{\min }+\mathbf{b}^{\prime}\left(\mathcal{A}_{u}^{\prime}\right)$, respectively.

Condition 3. $\left(V\left(\mathcal{A}_{u}\right) \cup V\left(\mathcal{A}_{u}^{\prime}\right)\right) \cap T(E)=\emptyset$. 
Condition 4. $X\left(\mathcal{A}_{u}, \mathcal{A}_{u}^{\prime}\right)$ is sufficient.

Condition 5. Both $\mathcal{A}_{u}$ and $\mathcal{A}_{u}^{\prime}$ are complete pushing sets.

The following theorem holds.

Theorem 5. $\left(\mathcal{A}_{u}, \mathcal{A}_{u}^{\prime}\right)$ is feasible if and only if Conditions 1 to 5 are met.

Proof. This proof consists of two parts. In the first part, we prove the necessity of these five conditions, whereas in the second part we prove the sufficiency of the combination of these five conditions. Note that as we mentioned earlier, a pair $\left(\mathcal{A}_{u}, \mathcal{A}_{u}^{\prime}\right)$ is feasible if its associated 3-tuple $\left(X\left(\mathcal{A}_{u}, \mathcal{A}_{u}^{\prime}\right), \mathbf{b}\left(\mathcal{A}_{u}\right), \mathbf{b}^{\prime}\left(\mathcal{A}_{u}\right)\right)$ induces $\left(\mathbf{s}, \mathbf{s}^{\prime}\right)$.

In the following, we provide reasons why the violation of each of Conditions 1 to 5 guarantees the infeasibility of $\left(\mathcal{A}_{u}, \mathcal{A}_{u}^{\prime}\right)$.

- If $i, i^{\prime}, j \in N$ such that $(j, i) \in \mathcal{A}_{u}$ and $\left(j, i^{\prime}\right) \in \mathcal{A}_{u}$, then whatever value $b_{j}\left(\mathcal{A}_{u}\right)$ takes we have

$$
s_{j}+p_{j}^{\min }+b_{j}\left(\mathcal{A}_{u}\right)=s_{i}=s_{i^{\prime}}
$$

A similar argument is true if $i, i^{\prime}, j \in N$ such that $(j, i) \in \mathcal{A}_{u}^{\prime}$ and $\left(j, i^{\prime}\right) \in \mathcal{A}_{u}^{\prime}$, for which case $s_{i}^{\prime}$ must be equal to $s_{i^{\prime}}^{\prime}$. Notice that any pair $\left(\mathcal{A}_{u}, \mathcal{A}_{u}^{\prime}\right)$ that violates Condition 1 contradicts the above arguments.

- If Condition 2 does not hold, then any 3-tuple $\left(X, \mathbf{b}, \mathbf{b}^{\prime}\right)$ such that $\mathcal{A}_{u} \subseteq \mathcal{A}(\mathbf{s}, X, \mathbf{b})$ and $\mathcal{A}_{u}^{\prime} \subseteq \mathcal{A}\left(\mathbf{s}^{\prime}, X, \mathbf{b}^{\prime}\right)$ cannot induce $\left(\mathbf{s}, \mathbf{s}^{\prime}\right)$ simply because the pushing pairs in $\mathcal{A}_{u}$ and $\mathcal{A}_{u}^{\prime}$ (which also exist in $\mathcal{A}(\mathbf{s}, X, \mathbf{b})$ and $\mathcal{A}\left(\mathbf{s}^{\prime}, X, \mathbf{b}^{\prime}\right)$, respectively) cause resource infeasibilities in schedule $\mathbf{s}$ and/or schedule $\mathbf{s}^{\prime}$.

- If Condition 3 does not hold, then the pushing pairs in $\mathcal{A}_{u}$ and $\mathcal{A}_{u}^{\prime}$ cause the violation of some of the precedence constraints in $T(E)$ and thus any 3 -tuple $\left(X, \mathbf{b}, \mathbf{b}^{\prime}\right)$ such that $\mathcal{A}_{u} \subseteq \mathcal{A}(\mathbf{s}, X, \mathbf{b})$ and $\mathcal{A}_{u}^{\prime} \subseteq \mathcal{A}\left(\mathbf{s}^{\prime}, X, \mathbf{b}^{\prime}\right)$ cannot induce $\left(\mathbf{s}, \mathbf{s}^{\prime}\right)$.

- Condition 4 must not be violated because otherwise no selection $X \subseteq X\left(\mathcal{A}_{u}, \mathcal{A}_{u}^{\prime}\right)$ is sufficient and thus $\left(\mathbf{s}, \mathbf{s}^{\prime}\right)$ cannot be induced.

- Both $\mathcal{A}_{u}$ and $\mathcal{A}_{u}^{\prime}$ must be complete pushing sets because otherwise based on Theorem 4 we have $\mathbf{s} \neq E S\left(X\left(\mathcal{A}_{u}, \mathcal{A}_{u}^{\prime}\right), \mathbf{p}^{\mathrm{min}}+\mathbf{b}\left(\mathcal{A}_{u}\right)\right)$ and $/$ or $\mathbf{s}^{\prime} \neq E S\left(X\left(\mathcal{A}_{u}, \mathcal{A}_{u}^{\prime}\right), \mathbf{p}^{\mathrm{min}}+\mathbf{b}^{\prime}\left(\mathcal{A}_{u}\right)\right)$.

In the second part of the proof, we provide adequate reasonings that support the sufficiency of the combination of the above five conditions to conclude the feasibility of $\left(\mathcal{A}_{u}, \mathcal{A}_{u}^{\prime}\right)$. When Conditions 1 to 4 hold, we can infer that both schedules resulting from $E S\left(X\left(\mathcal{A}_{u}, \mathcal{A}_{u}^{\prime}\right), \mathbf{p}^{\min }+\mathbf{b}\left(\mathcal{A}_{u}\right)\right)$ and $E S\left(X\left(\mathcal{A}_{u}, \mathcal{A}_{u}^{\prime}\right), \mathbf{p}^{\text {min }}+\mathbf{b}^{\prime}\left(\mathcal{A}_{u}\right)\right)$ are stochastically semi-active (because of Condition 1 ), resource feasible (because of Conditions 2 and 4) and precedence feasible (because of Condition 3). When Condition 5 holds, based on Theorem 4 we have

$$
\begin{aligned}
\mathbf{s} & =E S\left(X\left(\mathcal{A}_{u}, \mathcal{A}_{u}^{\prime}\right), \mathbf{p}^{\min }+\mathbf{b}\left(\mathcal{A}_{u}\right)\right) \text { and } \\
\mathbf{s}^{\prime} & =E S\left(X\left(\mathcal{A}_{u}, \mathcal{A}_{u}^{\prime}\right), \mathbf{p}^{\min }+\mathbf{b}^{\prime}\left(\mathcal{A}_{u}\right)\right) .
\end{aligned}
$$

Therefore, we conclude that Conditions 1 to 5 are sufficient to determine the feasibility of $\left(\mathcal{A}_{u}, \mathcal{A}_{u}^{\prime}\right)$. 
Each node $\left(\mathcal{A}_{u}, \mathcal{A}_{u}^{\prime}\right)$ in an even level $l$ (including level zero $(l=0)$ ) is branched into a number of nodes $\left(\mathcal{A}_{u} \cup\{(j, i)\}, \mathcal{A}_{u}^{\prime}\right)$ in level $l+1$ (which is an odd level) with associated activity $i=\mathrm{AL}_{\lceil(l+1) / 2\rceil}$ where $(j, i) \in T\left(E \cup X\left(\mathcal{A}_{u}, \mathcal{A}_{u}^{\prime}\right)\right)$. Likewise, each node $\left(\mathcal{A}_{u}, \mathcal{A}_{u}^{\prime}\right)$ in an odd level $l^{\prime}$ is branched into a number of nodes $\left(\mathcal{A}_{u}, \mathcal{A}_{u}^{\prime} \cup\left\{\left(j^{\prime}, i^{\prime}\right)\right\}\right)$ in level $l^{\prime}+1$ (which is an even level) with an associated activity $i^{\prime}=\mathrm{AL}_{\left\lceil\left(l^{\prime}+1\right) / 2\right\rceil}$ where $\left(j^{\prime}, i^{\prime}\right) \in T\left(E \cup X\left(\mathcal{A}_{u}, \mathcal{A}_{u}^{\prime}\right)\right)$.

Theorem 6. The violation of each one of Conditions 1 to 4 in node $\left(\mathcal{A}_{u}, \mathcal{A}_{u}^{\prime}\right)$ guarantees that no direct or transitive child of $\left(\mathcal{A}_{u}, \mathcal{A}_{u}^{\prime}\right)$ is feasible. Therefore, such a node must be fathomed.

Proof. The proof becomes apparent by referring to the first four bullets in the proof of Theorem 5 .

The branching starts with the root node $\mathcal{N}_{0}:\left(\mathcal{A}_{0}, \mathcal{A}_{0}^{\prime}\right)=(\emptyset, \emptyset)$. The root node is branched into a number of nodes $(\{(j, i)\}, \emptyset)$, each associated with one possible pushing pair $(j, i)$ to be added to $\mathcal{A}_{u}$. In the second level, each node $(\{(j, i)\}, \emptyset)$ is branched into a number of nodes $\left(\{(j, i)\},\left\{\left(j^{\prime}, i\right)\right\}\right)$, each associated with one possible pushing pair $\left(j^{\prime}, i\right)$ to be added to $\mathcal{A}_{u}^{\prime}$. In the other levels we continue branching in the same fashion as in the first two levels. Backtracking occurs if at least one of the Conditions 1 to 4 is violated (see Theorem 6). As soon as a pair of complete pushing sets is constructed (which happens when we visit a feasible node on the level $2(n-|B|+1)$ ), the algorithm immediately halts and the answer to problem BBP is 'YES'. If after visiting all nodes in the tree no such pair of complete pushing sets is constructed, the answer to problem BBP is 'NO'.

There are three main reasons that the order based on which we test the five conditions is not included in the text. The first reason is that they are overlapping (meaning that, e.g., parts of the process to test Condition 3 are done while testing Condition 2). The second reason is that such an order is of little importance since we noticed that the runtime of the algorithm is mainly affected by the exponential number of nodes in the tree and certainly not by the order of testing these five conditions. And finally, the third reason is that the main purpose of this research is to emphasize the importance of buffer-based reactions versus non-selection-based and selection-but-not-buffer-based reactions and not to evaluate the computational efficiency of a given sub-procedure that solves a subproblem whose computational complexity is still open.

Example. Consider the selection-based reaction from $\mathbf{s}^{7}$ and $\mathbf{s}^{9}$ for the slightly changed instance provided in Section 3.2. In order to determine whether this reaction is also buffer-based or not, we use the algorithm described above to implicitly enumerate all potential 3-tuples $\left(X, \mathbf{b}^{7}, \mathbf{b}^{9}\right)$.

We construct $A L$ by ordering activities based on their starting times in one of the schedules (in this case schedule $\mathbf{s}^{7}$ ) and removing all activities that start at time zero (these activities are pushed by the dummy start activity):

$$
\mathrm{AL}=(3,6,2,5,7,8,9) .
$$

We also compute $X=\left(\mathcal{X}_{\mathbf{s}^{7}, \mathbf{0}} \cap \mathcal{X}_{\mathbf{s}^{9}, \mathbf{0}}\right) \backslash T(E)$ as follows:

$$
X=\{(1,2),(1,6),(1,7),(1,8),(3,6),(3,7),(3,8),(4,3),(4,5)\} .
$$

Figure 6 illustrates the tree associated with this example. The label above each node indicates the pushing pair that is added to the partially constructed pushing sets. The number inside each node represents the index of the node (notice that nodes are visited in the non-decreasing order of their indices). The associated activity in level one and level two is $\mathrm{AL}_{\lceil 1 / 2\rceil}=\mathrm{AL}_{\lceil 2 / 2\rceil}=3$, the associated activity in level three and level four is $\mathrm{AL}_{\lceil 3 / 2\rceil}=\mathrm{AL}_{\lceil 4 / 2\rceil}=6$ and the associated activity 


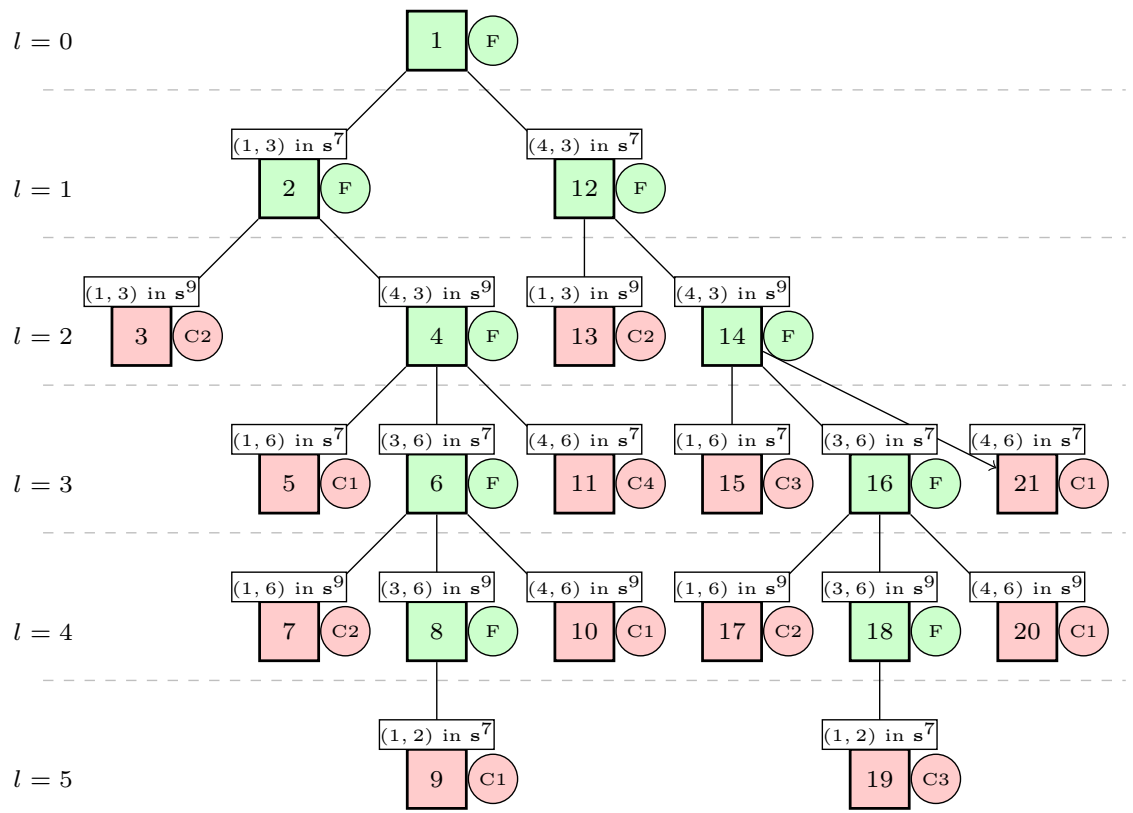

Figure 6: The tree associated with the example in Section 4.

in level five is $\mathrm{AL}_{[5 / 2\rceil}=2$. The branching starts with the root node $\mathcal{N}_{1}:\left(\mathcal{A}_{1}^{7}, \mathcal{A}_{1}^{9}\right)=(\emptyset, \emptyset)$ in level zero. The root node is branching into two nodes: the first child node $\mathcal{N}_{2}:\left(\mathcal{A}_{2}^{7}, \mathcal{A}_{2}^{9}\right)=(\{(1,3)\}, \emptyset)$ enforces activity 3 to be pushed by activity 1 in $\mathbf{s}^{7}$ and the second child node $\mathcal{N}_{12}:(\{(4,3)\}, \emptyset)$ enforces activity 3 to be pushed by activity 4 in $\mathbf{s}^{7}$. Likewise, $\mathcal{N}_{2}$ is branched into two nodes: the first child node $\mathcal{N}_{3}:(\{(1,3)\},\{(1,3)\})$ enforces activity 3 to be pushed by activity 1 in both $\mathbf{s}^{7}$ and $\mathbf{s}^{9}$ whereas the second child node $\mathcal{N}_{4}:(\{(1,3)\},\{(4,3)\})$ enforces activity 3 to be pushed by activity 1 in $\mathbf{s}^{7}$ and by activity 4 in $\mathbf{s}^{9}$. All other nodes are branched in the same fashion.

All nodes with an ' $F$ ' letter are associated with feasible partially constructed sets of pushing pairs. All nodes with a ' $C$ ' letter are nodes that are infeasible due to one of the conditions discussed above. For instance, node $\mathcal{N}_{5}$ violates Condition 1 because activity 1 pushes both activities 3 and 6 in $\mathbf{s}^{7}$, yet $s_{3}^{7} \neq s_{6}^{7}$. Node $\mathcal{N}_{3}$ violates Condition 2 because $\mathbf{s}^{9}$ is not resource feasible for $\mathbf{p}^{\min }+\mathbf{b}^{9}\left(\mathcal{A}_{3}^{9}\right)$ at time 2 when activities 1 and 4 are ongoing and there are not enough available resources to start activity 2. Node $\mathcal{N}_{15}$ violates Condition 3 because $\left(V\left(\mathcal{A}_{15}^{7}\right) \cup V\left(\mathcal{A}_{15}^{9}\right)\right) \cap T(E)=\{(1,3)\}$. Finally, node $\mathcal{N}_{11}$ violates Condition 4 because

$$
\begin{aligned}
X\left(\mathcal{A}_{11}^{7}, \mathcal{A}_{11}^{9}\right) & =X^{a} \backslash\{(4,3)\} \\
& =\{(1,2),(1,6),(1,7),(1,8),(3,6),(3,7),(3,8),(4,5)\}
\end{aligned}
$$

is not sufficient (there exists a forbidden set $\{2,3,4\}$ ). As after visiting all nodes in the tree, no pair of complete pushing sets is constructed, we conclude that the answer to problem BBP is ' $N O$ ' and thus the reaction from $\mathbf{s}^{7}$ to $\mathbf{s}^{9}$ is not buffer-based. 


\begin{tabular}{c|c|cccc}
$w_{b}$ & $w_{r}$ & SB & SBS & SBO & $\frac{\text { SBO }}{\text { SB }}$ \\
\hline \hline \multirow{3}{*}{25} & 0 & 48.57 & 94.34 & 98.87 & 2.04 \\
& 50 & 48.57 & 92.30 & 98.21 & 2.02 \\
& 100 & 48.57 & 90.00 & 97.67 & 2.01 \\
\hline \multirow{3}{*}{50} & 0 & 48.57 & 94.34 & 98.69 & 2.03 \\
& 50 & 48.57 & 92.30 & 98.69 & 2.03 \\
& 100 & 48.57 & 90.00 & 98.38 & 2.03
\end{tabular}

Table 3: The ratio (in percentage) of the selection-based reactions to all reactions.

\section{Computational results}

In this section, we run an experiment to evaluate the importance of selection-based reactions and buffer-based reactions. In this experiment, we use Model 3 proposed in Davari and Demeulemeester (2017) to obtain a PR-policy. We set $w_{j, k}=w_{j, 0}$ for every $j \in N$ and $\kappa_{1}=500, \kappa_{2}=50$ and $\kappa_{3}=4$ $\left(\kappa_{1}, \kappa_{2}\right.$ and $\kappa_{3}$ are related to the pool/schedule generation procedure in Davari and Demeulemeester (2017) and the assigned values are chosen based on some preliminary experiments.) Model 3 has been implemented in Visual $\mathrm{C}++2010$. All computational results were obtained on a laptop Dell Latitude with $2.6 \mathrm{GHz}$ Core(TM) i7-3720QM processor, 8GB of RAM and running under Windows 10.

In Table 3, we report the ratio (in percentage) of the selection-based reactions to all reactions (SB), the ratio (in percentage) of selected selection-based reactions to all selected reactions (SBS), the ratio (in percentage) of selection-based reactions in the optimal PR-policy to all reactions in the optimal PR-policy (SBO) and the ratio $\frac{\mathrm{SBO}}{\mathrm{SB}}$ for different choices of $w_{b}$ and $w_{r}$. The ratio $\frac{\mathrm{SBO}}{\mathrm{SB}}$ indicates how much larger SBO is than SB, which essentially also indicates the importance of selection-based reactions in the optimal PR-policy. In our experiments, the ratio $\frac{\mathrm{SBO}}{\mathrm{SB}}$ is always greater than one which means that SBO is greater than SB and therefore implicitly emphasizes the importance of selection-based reactions versus non-selection-based reactions in constructing the optimal PR-policy. If $\frac{\mathrm{SBO}}{\mathrm{SB}}$ would have been less than or equal to one, then it would mean that SBO is less than or equal to SB and this would indicate that selection-based reactions are not more important than non-selection-based reactions. Note that a reaction that is selected to be the best among its rivals, might not be part of the optimal solution. We observe that about half of the reactions in the network are selection-based. The fact that this number is so high is probably because of the way we generate the set of schedules. The results in Table 3 indicate that more than 90 percent of the reactions that are selected and more than 97 percent of the reactions in the optimal PR-policy are selection-based. By looking at the ratio $\frac{\mathrm{SBO}}{\mathrm{SB}}$, we notice that the chance of having a selection-based reaction in the optimal policy is more than two times larger than the chance of having a selection-based reaction in the whole network. This fact emphasizes even further the importance of selection-based reactions and suggests that studying these reactions and then focusing on generating them is a promising way to find higher-quality PR-policies.

To just get an idea of how much focusing on selection-based reactions will benefit us, we slightly modify Model 3 where we remove all non-selection-based reactions from the network. This model is called SB-Model 3 and its associated combined cost is referred to as SBCC. Table 4 reports some results on the comparison between Model 3 and SB-Model 3. As is shown in the table, by allowing 


\begin{tabular}{c|c|ccc}
$w_{b}$ & $w_{r}$ & SBCC & CC & DEV \\
\hline \hline \multirow{3}{*}{25} & 0 & 1692.26 & 1691.87 & 0.03 \\
& 50 & 1820.07 & 1819.70 & 0.02 \\
& 100 & 1899.88 & 1899.39 & 0.03 \\
\hline \multirow{3}{*}{50} & 0 & 3278.01 & 3277.43 & 0.02 \\
& 50 & 3437.57 & 3436.95 & 0.02 \\
& 100 & 3557.78 & 3557.43 & 0.01
\end{tabular}

Table 4: The average combined cost when only selection-based reactions are considered (SBCC), that when all reactions are considered (CC) and the average deviation (in percentage) of SBCC from CC.

\begin{tabular}{c|c|cccc}
$w_{b}$ & $w_{r}$ & BB & BBS & BBO & $\frac{\text { BBO }}{\text { BB }}$ \\
\hline \hline & 0 & 34.90 & 73.35 & 88.92 & 2.55 \\
25 & 50 & 34.90 & 70.85 & 86.44 & 2.48 \\
& 100 & 34.90 & 68.90 & 85.35 & 2.45 \\
\hline \multirow{3}{*}{50} & 0 & 34.90 & 73.35 & 88.55 & 2.54 \\
& 50 & 34.90 & 70.85 & 88.09 & 2.52 \\
& 100 & 34.90 & 68.90 & 86.84 & 2.49
\end{tabular}

Table 5: The ratio (in percentage) of the buffer-based reactions to all reactions.

only selection-based reactions, we on average lose less than 0.03 percent in quality while we need to solve a much smaller network, which is quite promising.

Within the class of selection-based reactions, the buffer-based reactions seem to be even more important. In Table 5, we report the ratio (in percentage) of the buffer-based reactions to all reactions (BB), the ratio (in percentage) of selected buffer-based reactions to all selected reactions (BBS), the ratio (in percentage) of buffer-based reactions in the optimal PR-policy to all reactions in the optimal PR-policy (BBO) and the ratio $\frac{\mathrm{BBO}}{\mathrm{BB}}$ for different choices of $w_{b}$ and $w_{r}$. These results indicate that only around 35 percent of the reactions are buffer-based. Nevertheless, these reactions do have a considerably large contribution in the optimal PR-policy (85 to 90 percent). By comparing the ratios $\frac{\mathrm{BBO}}{\mathrm{BB}}$ and $\frac{\mathrm{SBO}}{\mathrm{SB}}$, we may infer that buffer-based reactions are slightly more important than selection-based reactions. However, this conclusion is not completely fair since a large percentage (around 70 percent) of selection-based reactions are also buffer-based.

Table 6 reports the ratio (in percentage) of the selection-but-not-buffer-based reactions (selectionbased reactions that are not buffer-based) to all reactions (SNB), the ratio (in percentage) of selected selection-but-not-buffer-based reactions to all selected reactions (SNBS), the ratio (in percentage) of selection-but-not-buffer-based reactions in the optimal PR-policy to all reactions in the optimal PR-policy (SNBO) and the ratio $\frac{\mathrm{SNBO}}{\mathrm{SNB}}$ for different choices of $w_{b}$ and $w_{r}$. Notice that the class of buffer-based reactions and the class of selection-but-not-buffer-based reactions combined construct the class of selection-based reactions. We observe that around 15 percent of all reactions are selection-but-not-buffer-based reactions. Although these reactions are selection-based, they have a relatively small contribution (around 10 to 13 percent) in the optimal PR-policy. By looking at ratios $\frac{\mathrm{BBO}}{\mathrm{BB}}$ and $\frac{\mathrm{SNBO}}{\mathrm{SNB}}$, we observe that buffer-based reactions are considerably more important than selection-but-not-buffer-based reactions: the chance of having a buffer-based reaction in the 


\begin{tabular}{c|c|cccc}
$w_{b}$ & $w_{r}$ & SNB & SNBS & SNBO & $\frac{\text { SNBO }}{\text { SNB }}$ \\
\hline \hline \multirow{2}{*}{25} & 0 & 13.67 & 21.00 & 9.95 & 0.73 \\
& 50 & 13.67 & 21.45 & 11.77 & 0.86 \\
& 100 & 13.67 & 21.10 & 12.32 & 0.90 \\
\hline \multirow{3}{*}{50} & 0 & 13.67 & 21.00 & 10.14 & 0.74 \\
& 50 & 13.67 & 21.45 & 10.60 & 0.76 \\
& 100 & 13.67 & 21.10 & 11.54 & 0.84
\end{tabular}

Table 6: The ratio (in percentage) of the selection-but-not-buffer-based reactions to all reactions.

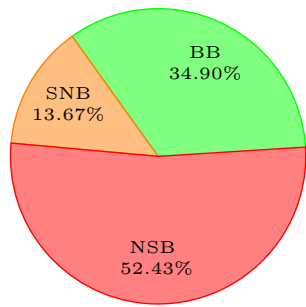

(a) The average contributions of different classes of reaction in the whole network

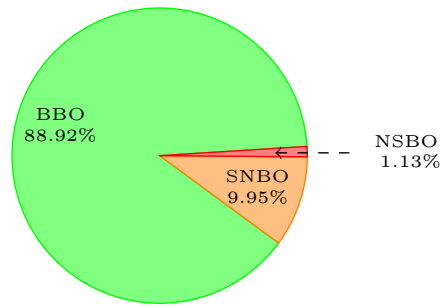

(b) The average contributions of different classes of reaction in the optimal PR-policy

Figure 7: The average contributions of different classes of reaction for the setting where $w_{b}=25$ and $w_{r}=0$.

optimal policy is almost 3 times larger than the chance of having a selection-but-not-buffer-based reaction in the optimal policy.

To further understand the importance of the buffer-based reactions, we report part of the information provided in Tables 3,5 and 6 using a pie chart representation. In Figure 7, we depict the contributions of three mutually exclusive and collectively exhaustive classes of reactions in the network of Model 3 and in its associated optimal PR-policy for the problem setting where $w_{b}=25$ and $w_{r}=0$.

The classes under comparison are the class of non-selection based (NSB) reactions, the class of buffer-based (BB) reactions and the class of selection-but-not-buffer-based (SNB) reactions. Figure 7 (a) shows the contributions of these classes of reactions in the whole network and Figure 7(b) displays the contributions of these classes of reactions in the associated optimal PR-policy. The futility of non-selection-based reactions in the optimal PR-policy is very clear in Figure 7 (NSBO represents the percentage of non-selection-based reactions in the optimal PR-policy): although 52.43 percent of the reactions are non-selection-based, the contribution of these reactions in the optimal PR-policy is only 1.13 percent. It is also clear that buffer-based reactions are very important: as stated before, despite the fact that only 34.90 percent of the reactions are buffer-based, their contribution in the optimal PR-policy is very high (88.92 percent).

To indicate how much we can rely solely on buffer-based reactions, we slightly modify Model 3 where we remove all non-buffer-based reactions from the network. This model is called BB-Model 3 and its associated combined cost is referred to as BBCC. Table 7 reports some results on the 


\begin{tabular}{c|c|ccc}
$w_{b}$ & $w_{r}$ & BBCC & CC & DEV \\
\hline \hline \multirow{3}{*}{25} & 0 & 1701.98 & 1691.87 & 0.67 \\
& 50 & 1826.28 & 1819.70 & 0.40 \\
& 100 & 1904.10 & 1899.39 & 0.27 \\
\hline \multirow{3}{*}{50} & 0 & 3288.60 & 3277.43 & 0.39 \\
& 50 & 3444.49 & 3436.95 & 0.24 \\
& 100 & 3563.02 & 3557.43 & 0.17
\end{tabular}

Table 7: The average combined cost when only buffer-based reactions are considered (BBCC), that when all reactions are considered (CC) and the average deviation (in percentage) of BBCC from CC.

\begin{tabular}{c|c|cccc} 
& & BB & BBS & BBO & $\frac{\text { BBO }}{\text { BB }}$ \\
\hline \hline \multirow{3}{*}{$\mathrm{NC}$} & 1.5 & 33.26 & 70.51 & 87.12 & 2.62 \\
& 1.8 & 32.83 & 68.92 & 85.30 & 2.60 \\
& 2.1 & 38.61 & 73.67 & 89.68 & 2.32 \\
\hline \multirow{4}{*}{$\mathrm{RF}$} & 0.25 & 50.01 & 77.67 & 90.42 & 1.81 \\
& 0.5 & 32.73 & 70.17 & 84.86 & 2.59 \\
& 0.75 & 25.51 & 68.78 & 88.28 & 3.46 \\
& 1 & 31.34 & 67.51 & 85.91 & 2.74 \\
\hline \multirow{3}{*}{$\mathrm{RS}$} & 0.2 & 19.07 & 80.70 & 94.27 & 4.94 \\
& 0.5 & 19.72 & 59.75 & 82.22 & 4.17 \\
& 0.7 & 28.91 & 63.22 & 84.87 & 2.94 \\
& 1 & 71.90 & 80.47 & 88.10 & 1.23
\end{tabular}

Table 8: The ratio (in percentage) of the buffer-based reactions to all reactions for different classes of instances.

comparison between Model 3 and BB-Model 3. As is shown in the table, by focusing only on buffer-based reactions, we lose on average between 0.17 to 0.67 percent in quality.

The importance of buffer-based reactions varies for different classes of instances. Table 8 reports the ratios $\mathrm{BB}, \mathrm{BBS}, \mathrm{BBO}$ and $\frac{\mathrm{BBO}}{\mathrm{BB}}$ for different values of $\mathrm{NC}$ (network complexity), for different values of RF (resource factor) and for different values of RS (resource strength). The parameter NC reflects the average number of non-redundant precedence relations per activity including dummy activities, the parameter RF represents the average portion of resources used and consumed and the parameter RS measures the strength of resource constrainedness. We refer interested readers to Kolisch and Sprecher (1997) where these parameters are introduced. Comparing the ratios $\mathrm{BB}, \mathrm{BBS}, \mathrm{BBO}$ and $\frac{\mathrm{BBO}}{\mathrm{BB}}$, we observe that the importance of buffer-based reactions remains almost constant when choosing different values for $\mathrm{NC}$. When $\mathrm{RF}$ is increased, the ratio $\mathrm{BBO}$ stays between 85 percent and 90 percent. However, the ratio BB first decreases (down to 25.51 percent) and then increases and therefore, $\frac{\mathrm{BBO}}{\mathrm{BB}}$ first increases (up to 3.446) and then decreases. When RS is increased, on the one hand, the ratio $\mathrm{BBO}$ first decreases (down to 82.22 percent) and then increases, and on the other hand, the ratio BB increases rapidly (specially when RS changes between 0.5 and 1 ). Taking both trends into consideration, we notice that by increasing RS the ratio $\frac{\mathrm{BBO}}{\mathrm{BB}}$ continuously decreases. All in all, we conclude that the buffer-based reactions are of the greatest importance when $\mathrm{RF}=0.75$ and $\mathrm{RS}=0.2$. 


\section{Discussion}

The results presented in Section 5 confirm the importance of the selection-based and buffer-based reactions, both of which put emphasis on the unique selection of resource arcs (resource flows) between schedules, in optimal PR-policies. One might wonder how and to what extent we can use this important property to construct a set of simple and compact rules that suggest proper reactions for infeasible situations without actually solving the PR-RCPSP. The idea of finding a set of simple and compact rules seems very similar to that of policies within the context of the stochastic resource-constrained project scheduling problem (SRCPSP). For a more detailed review of existing policies, we refer the interested readers to the first chapter of Davari (2017).

Following the results in the previous section, we observe that the probability that a reaction in an optimal PR-policy is selection-based is very high. This suggests that finding an appropriate selection based on which the reactions are made is very promising. Such a selection and its application very much resembles the set of added resource arcs associated with an early start policy (Radermacher, 1981; Stork, 2001). Let us introduce the class of early start PR-policies as follows: an early start PR-policy is represented by $\left(X, \mathbf{s}^{\text {base }}, \mathfrak{M}\right)$ where $X$ is a sufficient selection that also represents the additional resource arcs to be added to the precedence network, $\mathbf{s}^{\text {base }}$ represents a baseline schedule and $\mathfrak{M}$ is a mechanism that appropriately introduces buffers (which is crucially important when reaction costs are large). Aside from the process of finding $X$ and $\mathbf{s}^{\text {base }}$ that seems to be very difficult, developing $\mathfrak{M}$ appears to be very challenging. Therefore, what is discussed in this subsection is solely an attempt to introduce a future area of research.

Despite the fact that most of the reactions in an optimal PR-policy are selection-based, the number of unique associated selections in an optimal PR-policy can be very large and therefore searching for an early start PR-policy that is described in the previous paragraph seems to be very naive. Alternatively, we introduce another class of PR-policies. Consider a list $L$ of $|\mathcal{F}|$ items where each item is associated with one forbidden set in $\mathcal{F}$. List $L$ advocates a sufficient selection $X$ if for each $F S \in \mathcal{F}$ there exists a pair $(i, j) \in X$ such that $i, j \in F S$ and $j$ is the item in $L$ that is associated with $F S$. If one single list advocates all members of a set of selections, then we may argue that such a set of selections resembles a pre-selective policy (note that a pre-selective policy advocates a group of ES-policies that are slightly different from each other). Let us introduce the class of pre-selective PR-policies as follows: each pre-selective PR-policy is represented by $\left(L, \mathbf{s}^{\text {base }}, \mathfrak{M}\right)$. We believe that the following conjecture holds:

Conjecture 1. The following statements are true:

- An instance of SRCPSP can be converted to an instance of PR-RCPSP and can be optimally solved by any method that optimally solves PR-RCPSP. The optimal PR-policy associated with such an instance is the optimal policy to the SRCPSP.

- The counterparts of all classes of policies (in the context of SRCPSP) are subsets of the class of all PR-policies.

\section{$7 \quad$ Summary and conclusion}

In this paper, we introduce two very important classes of reactions, namely the class of selectionbased reactions and the class of buffer-based reactions. We show that the class of buffer-based reactions is a sub-class of the class of selection-based reactions and that selection-based reactions, 
specially those that are also buffer-based, contribute most in the construction of optimal PRpolicies. We also show that by relying only on selection-based reactions (or even by relying only on buffer-based reactions), we are still capable of producing high quality PR-policies.

Understanding and using these classes of reactions could pave a new avenue of research for interested researchers who wish to construct reasonably 'good' PR-polices without having to solve the PR-RCPSP. Moreover, we believe these two classes of reactions could be a starting point for those who plan to investigate other classes of reactions. We also encourage interested researchers to investigate the role of the classes of selection- and buffer-based reactions on the construction of the early-start and pre-selective PR-policies. Therefore, as a future research topic, we strongly advise to study the classes of early-start PR-policies and of pre-selective PR-policies and their potential links to the classes of selection- and buffer-based reactions. Interested researchers might also introduce other classes of PR-policies.

\section{References}

Alvarez-Valdes, R. and Tamarit, J. The project scheduling polyhedron: Dimension, facets and lifting theorems. European Journal of Operational Research, 67(2):204-220, 1993.

Artigues, C., Leus, R., and Talla Nobibon, F. Robust optimization for resource-constrained project scheduling with uncertain activity durations. Flexible Services and Manufacturing Journal, 25 (1-2):175-205, 2013.

Ashtiani, B., Leus, R., and Aryanezhad, M.-B. New competitive results for the stochastic resourceconstrained project scheduling problem: exploring the benefits of pre-processing. Journal of Scheduling, 14(2):157-171, 2011.

Ballestín, F. and Leus, R. Resource-constrained project scheduling for timely project completion with stochastic activity durations. Production and Operations Management, 18:459-474, 2009.

Ballestín, F. When is it worthwhile to work with the stochastic RCPSP? Journal of Scheduling, 10 (3):153-166, 2007.

Bertsekas, D. P. Dynamic Programming and Optimal Control. Athena Scientific, 2007.

Choi, J., Realff, M. J., and Lee, J. H. Dynamic programming in a heuristically confined state space: a stochastic resource-constrained project scheduling application. Computers $\& 3$ Chemical Engineering, 28(6):1039-1058, 2004.

Cong, J. Computing maximum weighted k-families and k-cofamilies in partially ordered sets. Technical report, University of California, 1993.

Creemers, S. Minimizing the expected makespan of a project with stochastic activity durations under resource constraints. Journal of Scheduling, 18(3):263-273, 2015.

Davari, M. Contributions to complex project and machine scheduling problems. PhD thesis, Faculty of Economics and Business, KU Leuven, 2017.

Davari, M. and Demeulemeester, E. A novel branch-and-bound algorithm for the chance-constrained RCPSP. Technical Report KBI_1620, KU Leuven, 2016. 
Davari, M. and Demeulemeester, E. The proactive and reactive resource-constrained project scheduling problem. Journal of Scheduling, 2017.

Fang, C., Kolisch, R., Wang, L., and Mu, C. An estimation of distribution algorithm and new computational results for the stochastic resource-constrained project scheduling problem. Flexible Services and Manufacturing Journal, 27(4):585-605, 2015.

Golumbic, M. C. CHAPTER 5 - comparability graphs. In Golumbic, M. C., editor, Algorithmic Graph Theory and Perfect Graphs, pages 105-148. Academic Press, 1980.

Grötschel, M., Lovász, L., and Schrijver, A. Polynomial algorithms for perfect graphs. In Berge, C. and Chvátal, V., editors, Topics on Perfect Graphs, volume 88 of North-Holland Mathematics Studies, pages 325-356. North-Holland, 1984.

Igelmund, G. and Radermacher, F. J. Preselective strategies for the optimization of stochastic project networks under resource constraints. Networks, 13(1):1-28, 1983.

Kaerkes, R. and Leipholz, B. Generalized network functions in flow networks. Methods of Operations Research, 27:441-465, 1977.

Kolisch, R. and Sprecher, A. PSPLIB - a project scheduling problem library. European Journal of Operational Research, 96(1):205-216, 1997.

Lambrechts, O., Demeulemeester, E., and Herroelen, W. Proactive and reactive strategies for resource-constrained project scheduling with uncertain resource availabilities. Journal of Scheduling, 11(2):121-136, 2008.

Leus, R. The generation of stable project plans. PhD thesis, Department of applied economics, Katholieke Universiteit Leuven, Belgium, 2003.

Leus, R. Resource allocation by means of project networks: Complexity results. Networks, 58(1): 59-67, 2011a.

Leus, R. Resource allocation by means of project networks: Dominance results. Networks, 58(1): $50-58,2011 b$.

Li, H. and Womer, N. K. Solving stochastic resource-constrained project scheduling problems by closed-loop approximate dynamic programming. European Journal of Operational Research, 246 (1):20-33, 2015.

Mehta, S. and Uzsoy, R. Predictive scheduling of a job shop subject to breakdowns. IEEE Transactions on Robotics and Automation, 14:365-378, 1998.

Möhring, R., Radermacher, F., and Weiss, G. Stochastic scheduling problems I - Set strategies. Mathematical Methods of Operations Research, 28:193-260, 1984.

Möhring, R., Radermacher, F., and Weiss, G. Stochastic scheduling problems II - set strategies. Mathematical Methods of Operations Research, 29(3):65-104, 1985.

Neumann, K., Schwindt, C., and Zimmermann, J. Project scheduling with time windows and scarce resources. Springer, 2003. 
Radermacher, F. Cost-dependent essential systems of es-strategies for stochastic scheduling problems. Methods of Operations Research, 42:17-31, 1981.

Rostami, S., Creemers, S., and Leus, R. New benchmark results for the stochastic resourceconstrained project scheduling problem. Journal of Scheduling, to appear, 2017.

Schwindt, C. Resource Allocation in Project Management. Springer, 2005.

Stork, F. Stochastic resource-constrained project scheduling. PhD thesis, Technical University of Berlin, School of Mathematics and Natural Sciences, 2001.

Van de Vonder, S., Ballestín, F., Demeulemeester, E., and Herroelen, W. Heuristic procedures for reactive project scheduling. Computers $\mathscr{E}$ Industrial Engineering, 52(1):11-28, 2007.

Van de Vonder, S., Demeulemeester, E., and Herroelen, W. Proactive heuristic procedures for robust project scheduling: An experimental analysis. European Journal of Operational Research, 189(3):723-733, 2008. 